ARTICLE

\title{
Mechanism of membrane-curvature generation by ER-tubule shaping proteins
}

\author{
Ning Wang ${ }^{1}$, Lindsay D. Clark (10 1, Yuan Gao ${ }^{1}$, Michael M. Kozlov (D) ${ }^{2}$, Tom Shemesh ${ }^{3}$ \& Tom A. Rapoport (D) ${ }^{1 凶}$
}

The endoplasmic reticulum (ER) network consists of tubules with high membrane curvature in cross-section, generated by the reticulons and REEPs. These proteins have two pairs of trans-membrane (TM) segments, followed by an amphipathic helix (APH), but how they induce curvature is poorly understood. Here, we show that REEPs form homodimers by interaction within the membrane. When overexpressed or reconstituted at high concentrations with phospholipids, REEPs cause extreme curvature through their TMs, generating lipoprotein particles instead of vesicles. The APH facilitates curvature generation, as its mutation prevents ER network formation of reconstituted proteoliposomes, and synthetic L- or D-amino acid peptides abolish ER network formation in Xenopus egg extracts. In Schizosaccharomyces japonicus, the APH is required for reticulon's exclusive ER-tubule localization and restricted mobility. Thus, the TMs and APH cooperate to generate high membrane curvature. We propose that the formation of splayed REEP/reticulon dimers is responsible for ER tubule formation.

\footnotetext{
${ }^{1}$ Howard Hughes Medical Institute and Department of Cell Biology, Harvard Medical School, Boston, MA 02115, USA. ${ }^{2}$ Department of Physiology and Pharmacology, Sackler Faculty of Medicine, Tel Aviv University, Tel Aviv 69978, Israel. ${ }^{3}$ Faculty of Biology, Technion-Israel Institute of Technology, Haifa 32000, Israel. ${ }^{凶}$ email: tom_rapoport@hms.harvard.edu
} 
ll cellular organelles have characteristic shapes, but how their morphology is generated is largely unknown. The endoplasmic reticulum (ER) is a particularly intriguing organelle, as it consists of morphologically distinct membrane domains, including a characteristic network of interconnected tubules $^{1,2}$. Interspersed in the network are sheets containing closely apposed membranes ${ }^{3}$. ER tubules have a diameter of $30-60 \mathrm{~nm}^{3,4}$ and thus have high membrane curvature in crosssection. A similar high curvature is seen at sheet edges ${ }^{3}$. The tubules themselves are shaped by two evolutionarily conserved protein families, the reticulons (Rtns) and REEPs (Yop1 in yeast $)^{5,6}$. In the absence of these proteins, tubules convert into sheets, but the tubular network formation can be restored when at least one of these proteins is expressed at a sufficient level ${ }^{3,5,6}$. When purified Rtn or REEP proteins are reconstituted into liposomes, they form short, narrow tubules ${ }^{7}$. Finally, these proteins localize exclusively to tubules and sheet edges, the high-curvature regions of the $\mathrm{ER}^{3,5,6}$.

Connecting tubules into a network requires membrane fusion, which is mediated by membrane-anchored GTPases, the Atlastins (ATLs) in metazoans and Sey1, and related proteins in yeast and plants $^{8-10}$. Proteoliposomes containing a purified fusion GTPase and a member of the reticulon/REEP families form a tubular membrane network in the presence of GTP, indicating that these are the minimal components to generate an ER network ${ }^{11}$. The ATLs also seem to be required for the formation of the tubules themselves, as depletion or inactivation of ATL causes the breakup of tubules into small vesicles or membrane fragments ${ }^{12}$.

The high membrane curvature of tubules and sheet edges is stabilized by the Rtns and REEPs by an unknown mechanism. All of these proteins contain pairs of closely spaced transmembrane (TM) segments followed by an amphipathic helix ${ }^{6,13,14}$ (APH; Fig. 1a and Supplementary Fig. 1a). It has been proposed that Rtns and REEPs generate high membrane curvature by hydrophobic insertion (wedging) and scaffolding 6,10 . Wedging would involve the shallow insertion of the APH into the cytoplasmic leaflet of the lipid bilayer, which would displace the polar head groups of the surrounding phospholipid molecules and thus produce local membrane bending ${ }^{6,13-16}$. Scaffolding would involve the formation of intrinsically curved arc-shaped protein oligomers on the membrane surface, which would mold the lipid bilayer into tubules. However, whether these mechanisms are responsible for the membrane-shaping activity of the Rtns and REEPs remains unclear. In this study, we use in vitro and in vivo experiments to show that the TMs and APH cooperate to generate high membrane curvature and to derive a model for ERtubule formation.

\section{Results}

REEP monomers form tightly interacting dimers within the membrane. The hydrophobic insertion mechanism of curvature generation can occur with protein monomers and does not require their oligomerization. By contrast, the formation of protein oligomers is essential for the scaffolding mechanism. To understand the mechanism by which REEPs and Rtns generate high membrane curvature, it is therefore important to determine their oligomeric states. As a model protein, we chose the REEP protein Yop1 from the fission yeast Schizosaccharomyces japonicus (referred to as Yop1), because this organism was used for in vivo experiments discussed in a later section. Yop1 was expressed in E. coli with a C-terminal streptavidin-binding peptide (SBP) tag and purified from the membrane fraction following solubilization in the detergent dodecylmaltoside (DDM). After affinity-chromatography on streptavidin beads and size-exclusion chromatography (SEC), the protein migrated as a single band of the expected size in Coomassie-blue stained SDS gels (Fig. 1b). It formed a dimer, as demonstrated by SEC combined with multiangle light scattering (MALS) (Fig. 1c). Similar results were obtained with Xenopus laevis REEP5, purified in the same way (Fig. 1b, c).

Dimer formation of Yop1 was confirmed with pull-down experiments. SBP-tagged full-length Yop1 (Yop1-SBP) or a mutant lacking the APH (Yop1 $\triangle \mathrm{APH}-\mathrm{SBP}$ ) were co-expressed in E. coli with similar versions of His-tagged Yop1 (Yop1-His10 or Yop1 $\mathrm{APH}-\mathrm{His} 10)$. DDM-solubilized membrane fractions were incubated with streptavidin beads, and the bound material was analyzed by SDS-PAGE and Coomassie-blue staining. The results show that the SBP-tagged protein pulled down the Histagged proteins, regardless of the presence of the APH (Fig. 1d). These results also indicate that dimer formation does not require the APH.

To further confirm dimerization, we inserted a folded protein, a variant of cytochrome b562 (BRIL) ${ }^{17}$, into the C-terminal segment of Yop1 (Supplementary Fig. 1b). The purified fusion protein was incubated with Fab fragments of BRIL antibodies ${ }^{18}$, and the sample was analyzed by negative-stain electron microscopy (EM) (Supplementary Fig. 1c-e). Most particles showed two dots corresponding to Fab fragments (Supplementary Fig. 1e), consistent with dimerization of the fusion protein. A mutant with a charge introduced into the hydrophobic side of the APH, Yop1 (I145R)-BRIL, also bound two Fab fragments (Supplementary Fig. 1e), indicating that the amphipathic nature of the helix is not required for dimerization.

Having shown Yop1 dimerization in detergent, we next tested whether dimer formation occurs in intact membranes. Photoreactive 4-benzoyl-L-phenylalanine (Bpa) residues were incorporated into different TMs of Yop1 by suppression of amber codons ${ }^{19,20}$. The purified proteins were irradiated with UV light either in DDM or after reconstitution into liposomes. Crosslinked products were analyzed by SDS-PAGE and Coomassieblue staining (Fig. 2a). Strong cross-linking was observed when Yop1 was reconstituted into membranes, and the probe was incorporated at F65 into TM2 (Yop1F65Bpa); $>75 \%$ of the monomers were converted into dimers. Probes in other TMs showed much less cross-linking (Fig. 2a). In detergent, all positions cross-linked only weakly, including F65 (Fig. 2a). We also incorporated photoreactive probes at many positions of the $\mathrm{APH}$, as well as positions preceding or following it, but none gave strong cross-links (Supplementary Fig. 2a, b), supporting the conclusion that the APH is not involved in dimerization. Incorporating Bpa into the N-terminal segment or the cytoplasmic loop between TMs 2 and 3 did not give strong cross-links either (Supplementary Fig. 2b). To confirm that cross-linking occurs between monomers in the dimer, rather than between dimers, we irradiated Yop1F65Bpa in liposomes and then solubilized the membranes in DDM and performed SEC. The cross-linked product eluted at the position of the dimer, rather than at a higher molecular weight (Supplementary Fig. 2c). Taken together, these results show that the REEP protein forms stable dimers, rather than monomers or higher oligomers, as previously assumed $^{6,21}$. The high cross-linking yields with a probe at F65 suggests that TM2 of two monomers tightly interact with one another inside the membrane. Further support for this assumption comes from experiments in which we placed the photoreactive probes at other positions in TM2 (Supplementary Fig. 2d). The cross-linking yields were lower but showed maxima at positions expected to be on one side of a helix formed by TM2 (positions 61, 65, and 69). These results suggest that dimerization is mediated by TM2. This TM is indeed special among the four TMs, as it contains well-conserved amino acids, including a Pro and several hydrophilic residues (Supplementary Fig. 2e). 
a

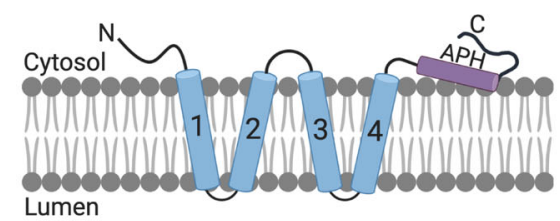

b

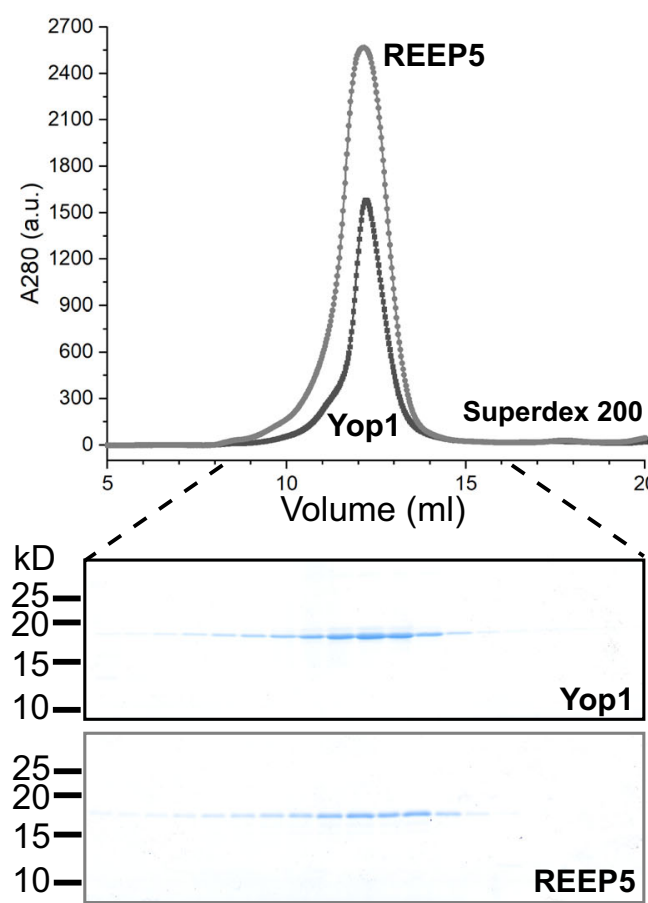

C

\begin{tabular}{c|c|c} 
& $\begin{array}{c}\text { Predicted Monomer } \\
\text { Size }(k D)\end{array}$ & $\begin{array}{c}\text { Calculated Protein } \\
\text { Mass (kD) }\end{array}$ \\
\hline $\begin{array}{c}\text { Xenopus } \\
\text { REEP5 }\end{array}$ & 22.4 & 47.7 \\
\hline $\begin{array}{l}\text { S. japonicus } \\
\text { Yop1-3C-SBP }\end{array}$ & 27 & 60.1 \\
\hline
\end{tabular}
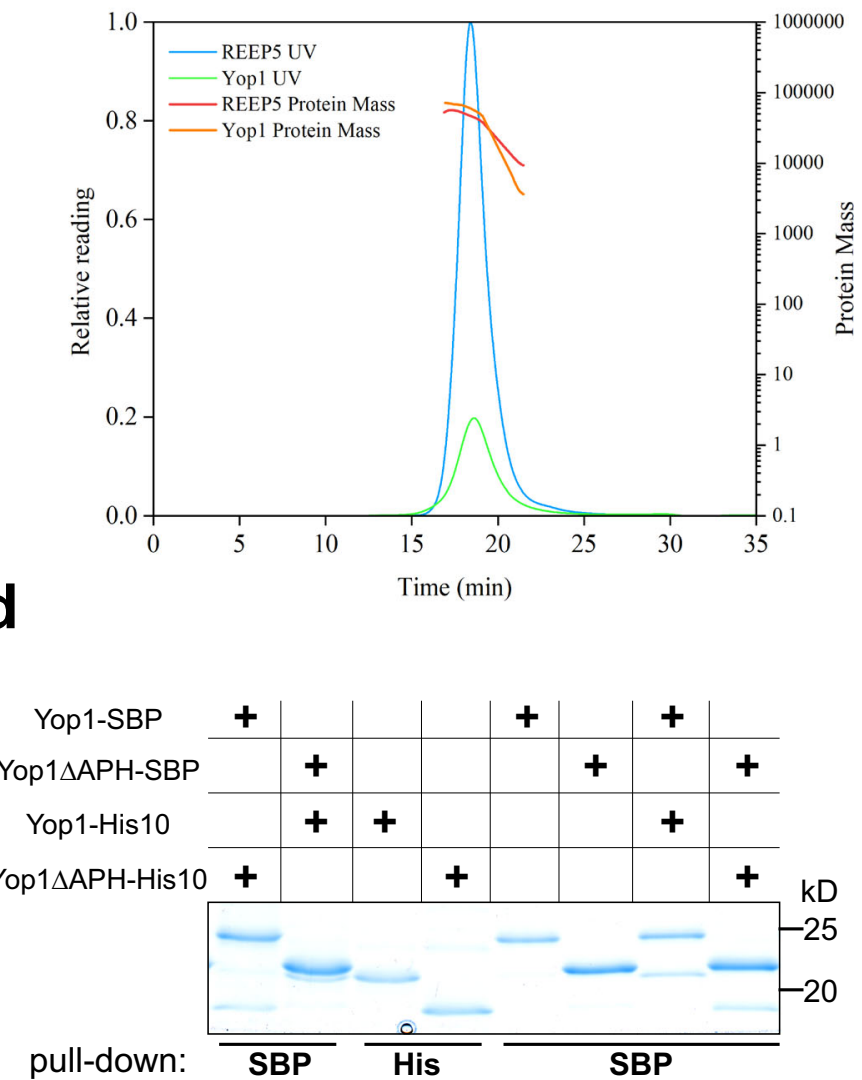

Fig. 1 REEPs form dimers. a Proposed membrane topology of the REEPs and Rtns. The four hydrophobic transmembrane segments (TMs) are numbered and shown in blue; the predicted amphipathic helix (APH) is shown in purple. b SEC of S. japonicus Yop1 and Xenopus laevis REEP5 purified in the detergent DDM. The experiment was performed four times. The top panel shows the absorbance at $280 \mathrm{~nm}$. Fractions of the eluate were analyzed by SDS-PAGE, and stained with Coomassie blue (lower panels). c SEC-MALS analysis of purified REEP5 and Yop1-3C-SBP, analyzed in DDM. Absorbance at $280 \mathrm{~nm}$ (UV) was normalized relative to the maximum reading of REEP5 (left axis). The calculated protein mass is shown across the peaks (right axis). The table on top shows the calculated and predicted monomer masses. d SBP-tagged full-length Yop1 or Yop1 lacking the APH (Yop1 1 APH) was co-expressed with Histagged full-length Yop1 or Yop1 $\triangle \mathrm{APH}$. DDM-solubilized membrane fractions were incubated with streptavidin beads and the bound material analyzed by SDS-PAGE and Coomassie-blue staining. As a control, SBP- and His-tagged Yop1 were individually expressed and purified. The experiment was performed three times.

Deletion of the APH or introduction of charged residues into the hydrophobic face of the helix reduced cross-linking of Yop1 with the probe at F65 (Fig. 2b; quantification see Fig. 2c). Thus, although the APH is not essential for dimerization of Yop1, it contributes to the interaction between the monomers in the membrane.

To test whether dimer formation is a general property of REEPs, we introduced a photoreactive probe at a position equivalent to F65 into Xenopus laevis REEP5, and into REEPs from two thermophilic fungi (Thielavia terrestris REEP5, and Thermothelomyces thermophila REEP5; Fig. 2d). In each case, irradiation of the purified, reconstituted protein gave dimer crosslinks, although not with the same efficiency as with Yop1. We conclude that the ground state of REEPs is a dimer in which monomers tightly interact within the membrane, an interaction facilitated by the APH. The reduced cross-linking efficiency in detergent suggests that dimers have a strained conformation in the membrane, which is relaxed upon solubilization.
Reconstituted REEP generates lipoprotein particles with extreme curvature. To investigate the membrane-shaping activity of REEPs, we inserted Yop1 into preformed liposomes (reconstitution by directed insertion ${ }^{22,23}$ ) and analyzed the resulting membrane structures by negative-stain EM. The generated structures were heterogeneous, likely because they contained Yop1 at different densities. The structures consisted of narrow short tubules (diameter 12-15 nm; length up to $600 \mathrm{~nm}$ ), small spherical particles with a diameter of $<12 \mathrm{~nm}$, vesicles with a diameter between 12 and $30 \mathrm{~nm}$, and vesicles with a diameter $>30 \mathrm{~nm}$ (Fig. 3a; quantifications in Fig. 3b). The small particles (diameter $<12 \mathrm{~nm}$ ) were observed previously with S. cerevisiae Yop1 or Rtn1, but were believed to be vesicles ${ }^{7}$. However, some of these particles have diameters smaller than $8 \mathrm{~nm}$, which makes it unlikely that they consist of a lipid bilayer surrounding an aqueous lumen (a bilayer has a thickness of $\sim 4 \mathrm{~nm}$ ). Rather, these structures appear to be lipoprotein particles (LPPs), i.e., protein/lipid micelles. Given their small diameter, the curvature of these structures is extremely high. When Yop1 lacking 
a
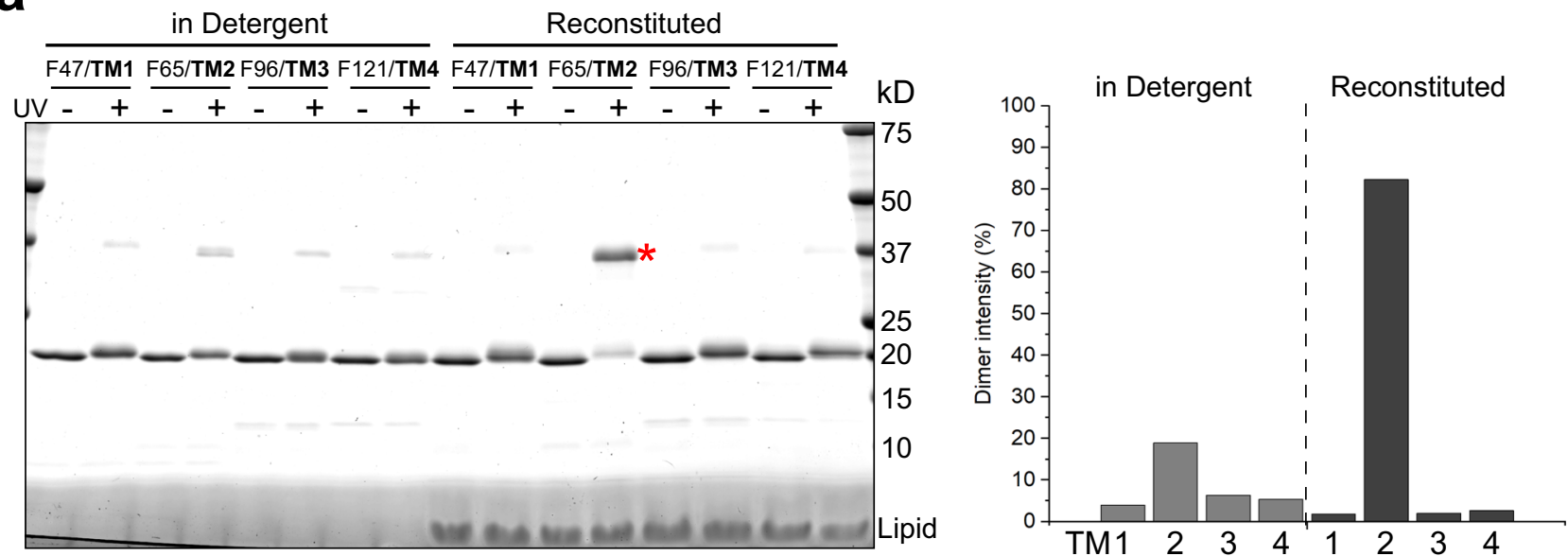

b

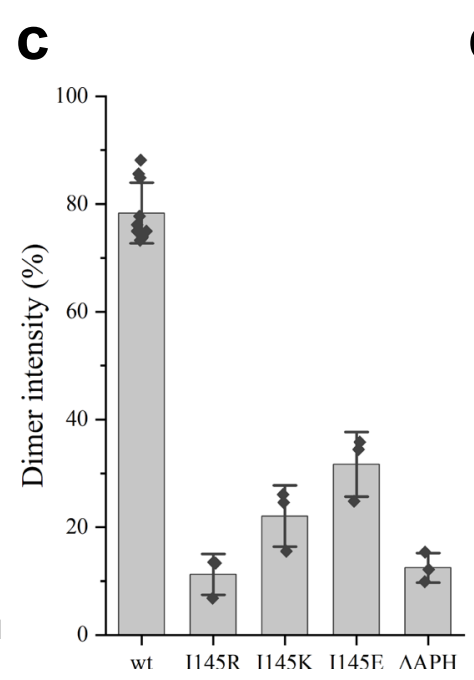

d
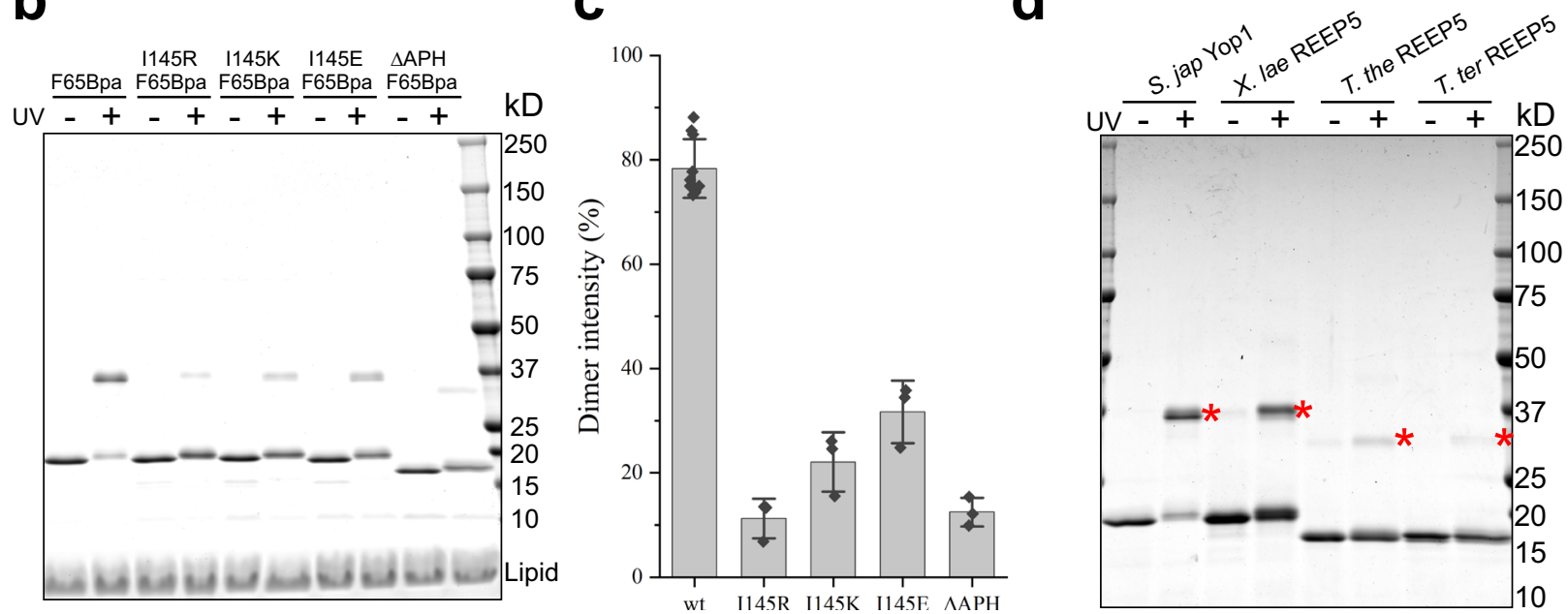

Fig. 2 REEP/Yop1 monomers interact in membranes through TM2. a Photoreactive Bpa probes were incorporated into Yop1 at the indicated positions of different TMs. The purified protein was irradiated with UV light either in DDM or after reconstitution in liposomes containing S. cerevisiae lipids at a 1:200 molar ratio of protein to lipid. The samples were analyzed by SDS-PAGE and Coomassie-blue staining. The asterisk indicates the position of the crosslinked Yop1F65Bpa dimer. The right panel shows quantification of the intensity of dimer cross-links relative to total protein. $\mathbf{b}$ As in $\mathbf{a}$, but with mutants in the $\mathrm{APH}$ reconstituted into proteoliposomes. c Quantification of experiments as carried out in $\mathbf{b}$. Data are presented as mean \pm the standard deviation (SD) from $n=10$ (wt) and $n=3$ (I145R, I145K, I145E, $\Delta \mathrm{APH}$ ) independent experiments. d Dimer formation was tested with purified REEPs from Schizosaccharomyces japonicus (S. jap), Xenopus laevis (X. lae), Thermothelomyces thermophila (T. the) and Thielavia terrestris ( $T$. ter). In each case, a Bpa probe was incorporated at the position of the conserved Phe residue in TM2. The samples were analyzed as in $\mathbf{a}$. The asterisks show the positions of cross-linked dimers.

the APH was reconstituted with phospholipids, tubules were almost absent, as previously reported ${ }^{13}$, and larger vesicles became more abundant (Fig. 3a, b). The number of LPPs decreased moderately (Fig. 3b). This suggests that LPPs were formed by domains of high Yop1 concentration, whereas domains of low Yop1 concentration required the APH to produce the curvature of tubules and/or small vesicles. When reconstituted wild-type Yop1 was subjected to flotation in a Nycodenz gradient (Fig. 3c), tubules and vesicles were found in the top fractions (Fig. 3d; F2), whereas the LPPs stayed near the bottom of the gradient (F4 + F5). Taken together, these results indicate that the TMs are largely responsible for producing the extreme curvature of LPPs generated at high protein concentrations. At lower Yop1 concentrations, the deletion of the APH leads to the conversion of tubules into larger vesicles.

Next, we performed similar experiments with Xenopus REEP5. Interestingly, this protein caused even higher membrane curvature than Yop1, generating mostly LPPs and a few larger vesicles, but essentially no tubules (Fig. 3e). In a flotation experiment, the bottom fractions consisted almost entirely of LPPs (Fig. 3f; F4 + F5; flotation pattern see Fig. 3c). In the top fractions, larger vesicles were seen from which LPPs appear to bud off (Fig. 3f; F2). Thus, Xenopus REEP5 seems to be more efficient than Yop1 in generating LPPs. This conclusion was confirmed with reconstitution experiments using different protein to lipid ratios, in which the samples were analyzed by negative-stain EM and flotation (Supplementary Fig. 3a). Xenopus REEP5 generated LPPs at even a 1:4000 protein to lipid ratio (Supplementary Fig. 3a), whereas Yop1 required considerably higher concentrations (1:80; Supplementary Fig. 3a). Xenopus REEP5 $\triangle \mathrm{APH}$ also generated LPPs at high concentrations (Supplementary Fig. 3b, c). Together, these results show that the REEPs can generate extreme curvature, but the efficiency depends on the species and concentration. For both Yop1 and Xenopus REEP5, the APH facilitates LPP formation but is not absolutely essential. 
a

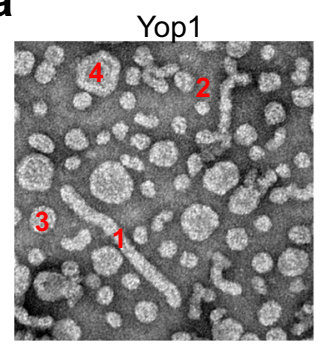

C

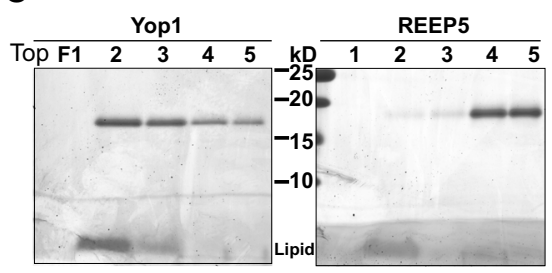

Yop1 $\triangle \mathrm{APH}$

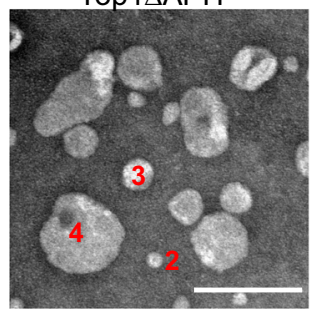

d b

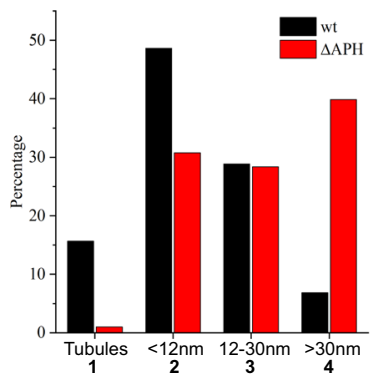

F2

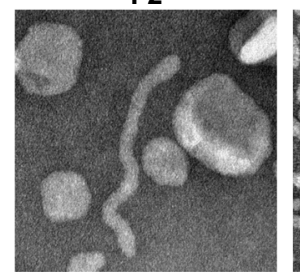

F2

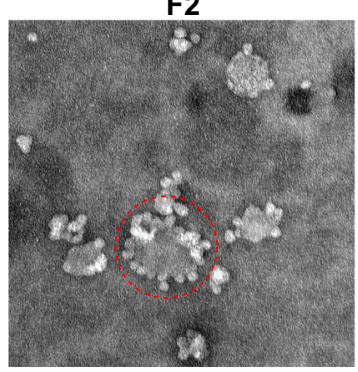

F4+F5

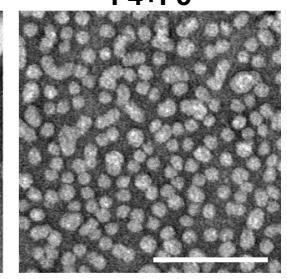

F4+F5

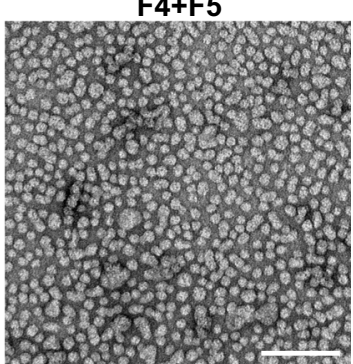

Fig. 3 Reconstitution of purified REEPs generates high-curvature structures. a Yop1 or Yop1 $\triangle$ APH were purified from DDM-solubilized membranes and reconstituted with S. cerevisiae lipids at a molar ratio of 1:200 protein:lipid. The samples were analyzed by negative-stain EM. Short tubules (1), vesicles with diameters $<12 \mathrm{~nm}$ (2), vesicles with diameters $12-30 \mathrm{~nm}$ (3), and vesicles with diameter $>30 \mathrm{~nm}$ (4) are highlighted. b Quantification of experiments as shown in a ( $n=2664$ for wt and 2632 for $\triangle$ APH from three independent experiments). c Yop1 or Xenopus REEP5 were reconstituted with S. cerevisiae lipids at a molar ratio of 1:200 protein:lipid. The samples were subjected to flotation in a Nycodenz gradient, and fractions were analyzed by SDS-PAGE and Coomassie-blue staining. The band at the bottom is lipid. $\mathbf{d}$ The indicated fractions of the Nycodenz flotation of Yop1 in $\mathbf{c}$ were analyzed by negative-stain EM. e Negative-stain EM analysis of Xenopus REEP5 after reconstitution, but before flotation. $\mathbf{f}$ Fractions of the Nycodenz flotation of Xenopus REEP5 in $\mathbf{c}$ were analyzed by negative-stain EM. Dashed circles in $\mathbf{e}$ and $\mathbf{f}$ highlight LPPs budding off from large proteoliposomes. All experiments were performed three times. Bars, $100 \mathrm{~nm}$.

REEPs generate LPPs in cells. Xenopus REEP5 could generate LPPs even when the protein was never exposed to detergent. When REEP5 was overexpressed in E. coli and the lysate subjected to centrifugation, a large fraction of the protein did not sediment with the membranes; up to $75 \%$ of the total population remained in the supernatant in LPPs (Fig. 4a, b). In contrast, Seyl sedimented almost quantitatively (Fig. 4a, b), as expected for an integral membrane protein. Deletion of the APH from REEP5 reduced LPP formation but did not abolish it (Fig. 4a), as in the reconstituted system (Supplementary Fig. 3b, c). Full-length REEP5 could be purified from the supernatant fraction without detergent, using the same protocol as for the material solubilized in detergent. After affinity-purification on streptavidin beads and SEC, essentially only one protein band was seen in a Coomassie-blue stained SDS gel (Fig. 4c, d). The protein had an apparent molecular weight of $\sim 400$ $\mathrm{kDa}$ in SEC. In negative-stain EM, the peak fractions contained almost spherical particles of relatively homogeneous size (inset of Fig. 4c; diameter 7-12 nm). Extraction with chloroform/methanol followed by thin-layer chromatography (TLC) indicated that these particles also contain lipids, mostly phosphatidylethanolamine (PE) and phosphatidylglycerol (PG) (Fig. 4e), a composition similar to that of E. coli membranes. Based on the molar ratio of REEP5 to phospholipid molecules (1:30), and the size of the particles, we estimate that each particle contains about 8 molecules of protein and 240 molecules of lipids. REEP5 in LPPs is fully functional, as it forms a GTP-dependent membrane network when co-reconstituted with the fusion GTPase Seyl ${ }^{11}$ (Fig. 4f). As expected, no lipid bilayer could be detected when the LPPs were analyzed by cryo-EM. In contrast, a bilayer was obvious in even small liposomes (diameters $<20 \mathrm{~nm}$; Supplementary Fig. 4a). These data show that Xenopus REEP5 generates the extreme membrane curvature of LPPs even without solubilization and reconstitution; it is simply the high concentration of REEP5 that converts phospholipid bilayers into LPPs.

To further estimate the number of REEP molecules per LPP, we inserted the folded BRIL protein into the cytosolic loop between TM2 and TM3 of Xenopus REEP5. Overexpressed REEP5-BRIL generated LPPs as efficiently in E. coli as wild-type REEP5 (Supplementary Fig. 4b). Again, the particles had a size range of 7-12 $\mathrm{nm}$ in negative-stain EM. The addition of Fabs directed against the BRIL protein showed particles with a variable number of bound Fab molecules (peak at 4-6 molecules; examples are shown in boxes of Supplementary Fig. 4b). Considering that not all bound Fab fragments are visible in these EM images, this number is consistent with our other estimate of the number of REEP molecules per particle. 
a

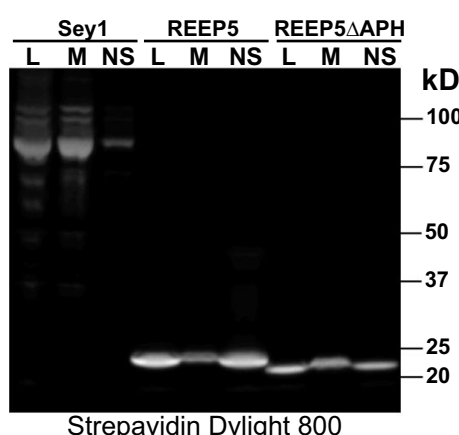

Strepavidin Dylight 800
C

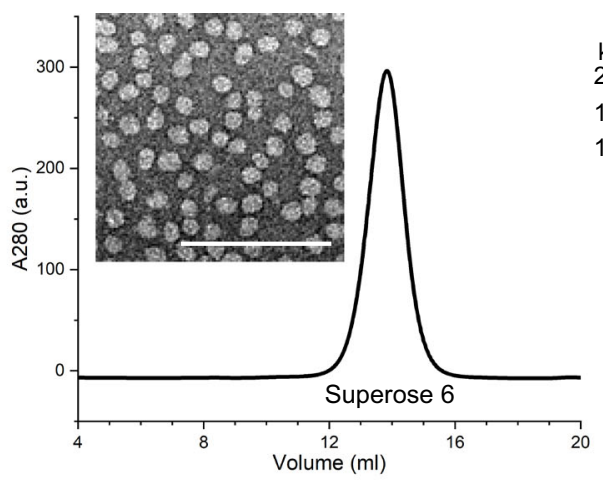

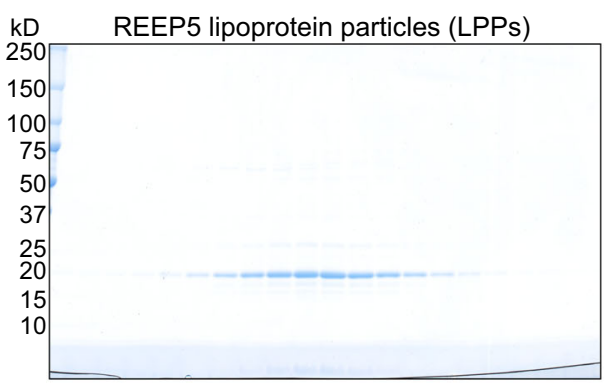

b

e

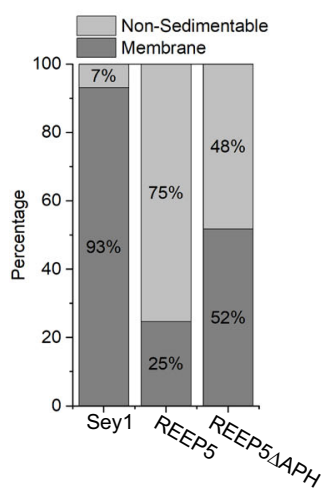

Lipid extracted from REEP5 LPPs

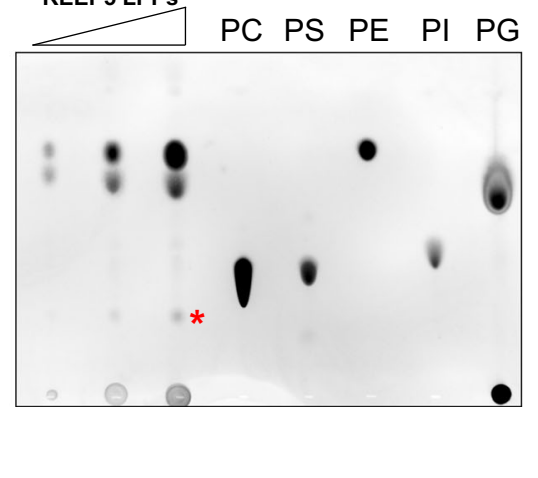

f
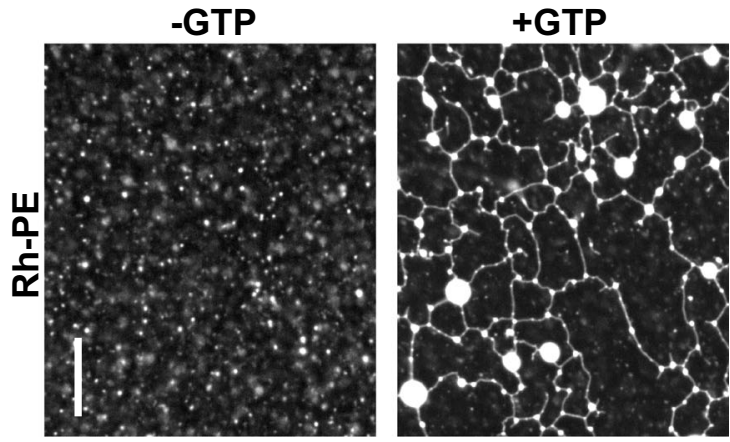

Fig. 4 Overexpression of REEP/Yop1 in E. coli generates lipoprotein particles (LPPs). a SBP-tagged REEP5, REEP5 $\Delta$ APH, or Sey1 were expressed in $E$. coli. Lysates (L) were fractionated by centrifugation into a non-sedimentable fraction (NS) and membrane fraction (M). Equivalent aliquots of these fractions were analyzed by SDS-PAGE, followed by blotting with Dylight 800-labeled streptavidin. b Quantification of the data in a. The experiment was performed two times. c Xenopus REEP5 was expressed in E. coli and purified from the NS fraction. It was then subjected to size-exclusion chromatography (SEC) and its elution followed by the absorbance at $280 \mathrm{~nm}$. The inset shows a representative negative-stain image of the peak fraction. The visualized structures are referred to as lipoprotein particles (LPPs). Bar, $100 \mathrm{~nm}$. d Fractions of the SEC run in c were analyzed by SDS-PAGE and Coomassie-blue staining. The experiment was performed four times. e Lipids were extracted from the purified LPPs, and different amounts of the extract were analyzed by thin-layer chromatography (TLC) and Primuline-staining. Pure PC, PS, PE, PI, and PG were run in parallel as markers. The asterisk indicates an unidentified lipid. The experiment was performed two times. f Purified LPPs were co-reconstituted with the GTPase Sey1 into liposomes containing POPC, DOPE, DOPS, and fluorescent rhodamine-PE (Rh-PE). The samples were incubated with or without GTP and visualized with a fluorescence microscope. The experiment was performed two times. Bar, $10 \mu \mathrm{m}$.

As with reconstituted proteins (Fig. 3), LPP formation in E. coli was less efficient with Yop1 than with Xenopus REEP5 (12\% versus $75 \%$ remaining in the supernatant after centrifugation; Supplementary Fig. 4c). We also tested LPP formation in E. coli with REEP5 proteins from T. thermophila and T. terrestris. Again, species differences were observed, with REEP5 from T. terrestris being considerably more active in LPP formation, despite being expressed at a lower level (33\% in the non-sedimentable fraction; Supplementary Fig. 4c). Negative-stain EM showed that the size of the LPPs formed by Yop1 and T. ter REEP5 was about the same as with Xenopus REEP5 (Supplementary Fig. 4d).

Interestingly, the interaction between the REEP monomers seems to be different in LPPs and membranes. We introduced a photoreactive probe into TM2 of Xenopus REEP5 or Yop1 and tested for dimer cross-links in the non-sedimentable LPP fraction and sedimentable membranes (Supplementary Fig. 4e). The nonsedimentable population reproducibly gave higher cross-linking yields, suggesting that most molecules are more tightly associated in LPPs than in low-curvature membranes. Taken together, these results indicate that REEPs generate LPPs when present at high concentrations, likely because REEP dimers generate so much curvature that they break up phospholipid bilayers into monolayers. The TMs seem to be the major factor for generating the high membrane curvature in LPPs, but the APH also contributes.

The APH is required to generate a tubular membrane network. To further analyze the role of the APH in curvature generation by the REEPs, we employed a reconstituted system in which the tubular ER network can be formed with liposomes containing purified proteins ${ }^{11}$. Full-length Xenopus REEP5 was reconstituted together with the fusion GTPase Seyl into proteoliposomes containing a hydrophobic fluorescent dye. Addition of GTP resulted in the formation of a tubular membrane network that could be visualized in a fluorescence microscope (Fig. 5a). No network was seen in the absence of GTP (Fig. 5a). Similar results were obtained with Yop1 (Fig. 5b). Xenopus REEP5 lacking the APH domain did not form a network (Fig. 5a), consistent with the postulated role of APH in generating high membrane curvature at endogenous protein concentrations ${ }^{11,14}$. Mutations in the hydrophobic side of Yop1's APH also reduced (I145S) or abolished (I145K) network formation (Fig. 5b). The APH of Yop1 
a
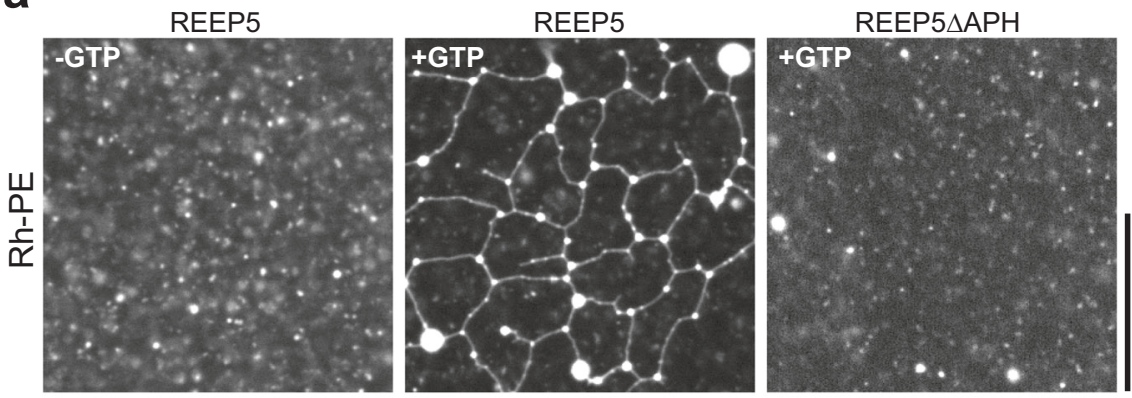

b
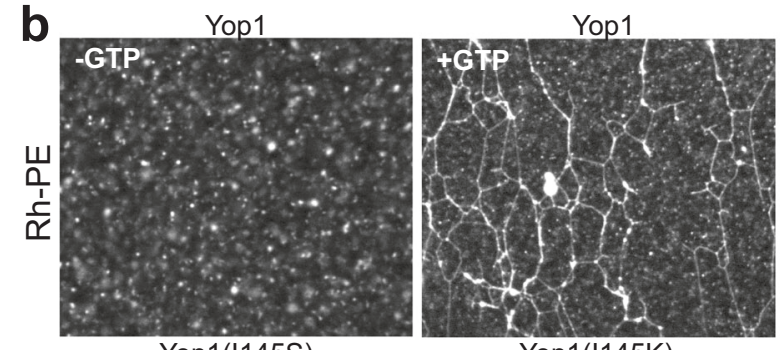

Yop1 $\triangle \mathrm{APH}$
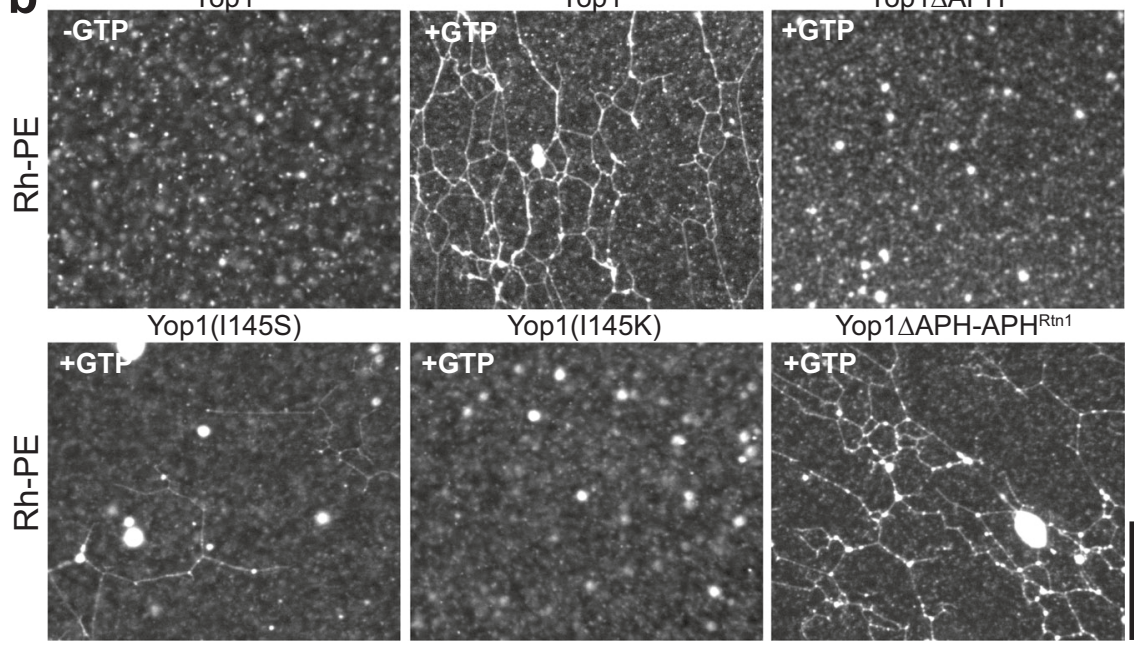

Fig. 5 The APH of REEPs is required for the reconstitution of a tubular network. a Xenopus REEP 5 or REEP5 lacking the APH (REEP5 $\triangle A P H)$ were purified from DDM-solubilized membranes and co-reconstituted with the GTPase Sey1 into liposomes containing POPC, DOPE, DOPS, and fluorescent Rh-PE. The samples were incubated with or without GTP, as indicated. The experiment was performed four times. b As in a, but with purified wild-type Yop1, a Yop1 mutant lacking the APH (Yop1 $\triangle \mathrm{APH})$, Yop1 variants with point mutations in the APH (Yop1(1145S) and Yop1(I145K)), or a Yop1 variant, in which the APH was replaced by that of sjRtn1 (Yop1 $\triangle \mathrm{APH}-\mathrm{APH}^{\mathrm{Rtn} 1}$ ). Bars (on the right), $10 \mu \mathrm{m}$. The experiment was performed two times.

could be replaced by that of $S$. japonicus Rtn1 (Fig. 5b), indicating that the APH plays a curvature-generating role in both tubuleshaping protein families.

The functional importance of the APH of REEPs was verified in vivo by analyzing the viability of $S$. cerevisiae cells. This organism was chosen because previous experiments had shown that the single or even combined deletion of the REEP and Rtn genes does not affect cell viability ${ }^{5}$, but a triple knockout of Rtn1, Yop1, and the nucleoporin Nup85 is lethal ${ }^{24}$. Expression of wildtype S. japonicus Yop1 or Xenopus REEP5 from a centromeric plasmid rescued the viability of this triple mutant, whereas expression of mutants lacking the APH or harboring point mutations in the APH did not (Supplementary Fig. 5a). Similar results were obtained with $S$. japonicus Rtn1: wild-type Rtn1 rescued the viability of the triple mutant, whereas APH mutants did not (Supplementary Fig. 5a).

Further evidence for APH generating high membrane curvature was obtained with synthetic peptides. A peptide containing the wild-type APH sequence of Yop1 bound to liposomes, as demonstrated by its flotation in a Nycodenz gradient (Fig. 6a). Circular dichroism (CD) spectrometry indicated that the peptide formed a helix when bound to liposomes, but was unstructured in buffer or DDM (Fig. 6b). An APH peptide carrying the I145K mutation had a reduced propensity to form a helix (Fig. 6b), although it still floated with the liposomes (Fig. 6a). When wild-type peptide was added to liposomes containing reconstituted Yop1, and the samples were analyzed by negative-stain EM, the abundance of LPPs increased and tubules and vesicles almost disappeared (Fig. 6c, d). In contrast, the I145K mutant peptide had little effect (Fig. 6c, d). Consistent with the increased number of LPPs, APH addition caused most of Yop1 to fractionate in heavy fractions of a Nycodenz gradient (Fig. 6e).

The APH can indeed contribute to the generation of excessive curvature that converts tubules into small membrane structures. This was demonstrated by adding a wild-type APH peptide of Yop1 to a reconstituted system containing both Yop1 and Sey1 (Fig. 7a). Instead of a membrane network, small vesicles and membrane fragments were observed that contained both lipid and protein (Fig. 7a and Supplementary Fig. 5b). In contrast, the I145K mutant peptide had no effect (Fig. 7a). A similar effect was seen when synthetic APH peptides were added to interphase Xenopus egg extracts that generate an ER network ${ }^{12,25}$. As in the reconstituted system, Yopl's wild-type APH peptide abolished the network (Fig. 7b-f), converting it into small membrane fragments (Fig. 7c). The I145K peptide was inactive (Fig. 7d). These results indicate that the amphipathic nature of the peptide is required to generate the high spontaneous curvature of the cytoplasmic membrane monolayer that breaks up the tubules into smaller membrane fragments.

A peptide corresponding to the APH of Xenopus REEP5 had the same effect as the Yop1 APH peptide, again converting the reticular ER network of interphase Xenopus egg extracts into small membrane fragments (Fig. 7e). To determine whether the APH promotes curvature by interaction with lipids or protein, we compared the effects of L- and D-amino acid APH peptides. We 
a

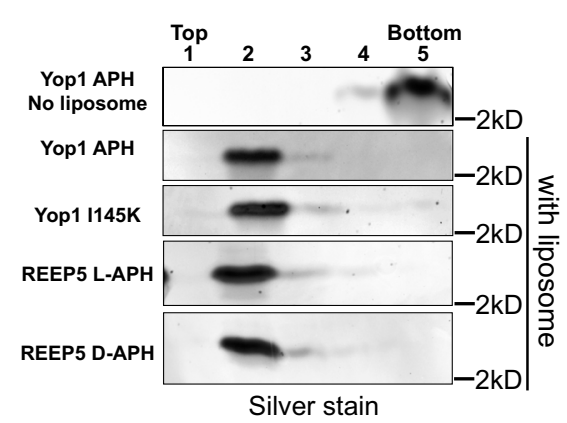

C

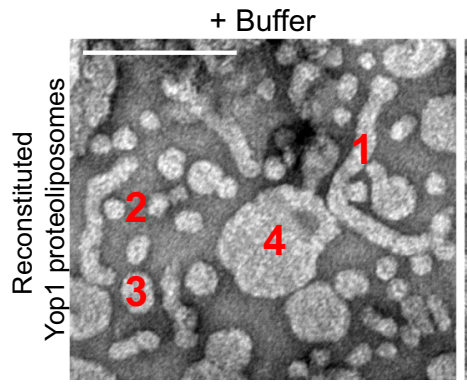

d

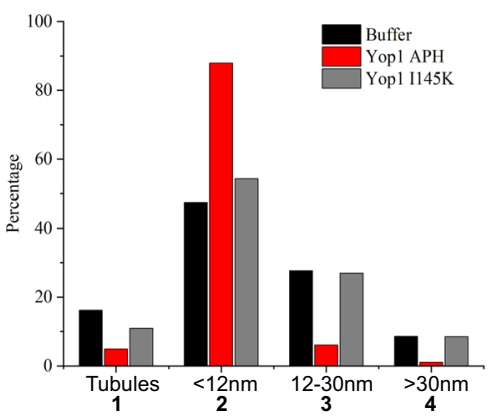

b

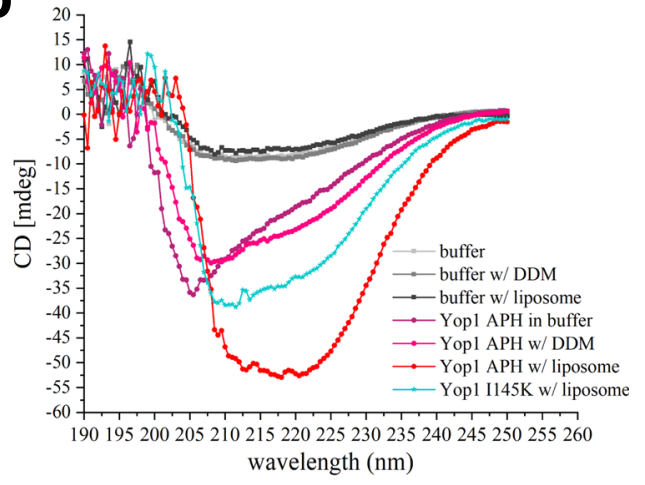

+ Yop1 APH

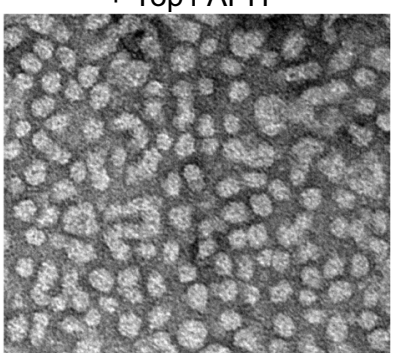

+ Yop1 I145K

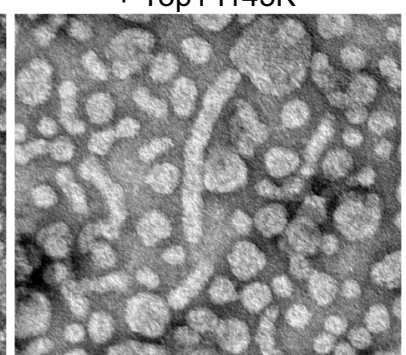

e

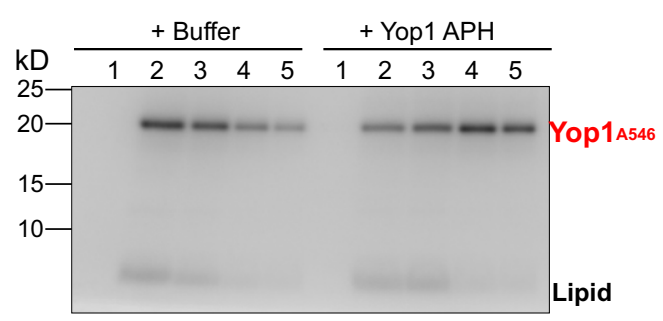

Fig. 6 Synthetic APH peptides bind to liposomes and generate high curvature. a Peptides corresponding to the APH of wild-type Yop1, to APH harboring a point mutation (Yop1 1145K), or to the APH of REEP5, with either L- or D-amino acids (REEP5 L-APH and REEP5 D-APH), were incubated with liposomes containing S. cerevisiae lipids at a 1:40 molar ratio of peptide to lipid. The samples were subjected to flotation in a Nycodenz gradient and analyzed by silver staining. The experiment was performed two times. The sequences of the peptides are shown in Supplementary Table 3. $\mathbf{b}$ The indicated peptides were analyzed by CD spectroscopy in the buffer, DDM, or after incubation with liposomes. The experiment was performed two times. $\mathbf{c}$ Yop1 was reconstituted into proteoliposomes and incubated with the indicated peptides. The samples were analyzed by negative-stain EM. Bar, $100 \mathrm{~nm}$. $\mathbf{d}$ Quantification of the structures seen in c. Short tubules (1), vesicles with diameters $<12 \mathrm{~nm}$ (2), vesicles with diameters 12-30 nm (3), and vesicles with diameter $>30 \mathrm{~nm}$ (4) were counted ( $n=1662$ for a buffer, 1490 for Yop1 APH, and 1800 for Yop1 I145K from two independent experiments). e Alexa 546-labeled Yop1 (Yop1 ${ }^{A 546}$ ) was reconstituted into liposomes containing S. cerevisiae lipids at a molar ratio of 1:200 protein to lipid. The samples were incubated with buffer or Yop1 peptide (1:20 molar ratio of peptide to lipid) and subjected to flotation in a Nycodenz gradient. Fractions were analyzed with a fluorescence scanner. The lower band corresponds to lipid. The experiment was performed three times.

found that a D-amino acid peptide of the same sequence as the Lamino acid peptide also disrupted the network (Fig. 7f). As expected, this peptide formed a helix that binds to liposomes (Fig. 6a), but it had the opposite chirality in CD spectrometry (Supplementary Fig. 5c). The L- and D-peptides also abolished a network generated with metaphase Xenopus egg extracts, which normally contains both tubules and small sheets ${ }^{12}$ (Fig. $7 g-i$ ). Given that both the L- and D-peptides convert tubules into structures of even higher membrane curvature, the APH likely acts by interaction with lipid, rather than protein. This is supported by experiments in which we added the L- and D-peptides to liposomes lacking proteins; negative-stain EM showed that large liposomes were converted into smaller vesicles or LPPs (Supplementary Fig. 5d).
Thus, at high concentrations, the APH alone can generate extreme curvature. Fractionation of the Xenopus extract, followed by immunoblotting for the endogenous REEP5 protein, showed that REEP5 is normally located entirely in sedimentable membranes, suggesting that LPPs do not form at physiological conditions. However, upon addition of the synthetic APH peptide, $15 \%$ of the total population remained in the supernatant, likely in LPPs, indicating that the APH can generate extreme curvature, even when the curvature-generating proteins are present at endogenous concentrations (Supplementary Fig. 5e, f).

We also tested the APH of Pex11, which had been implicated in tubule formation of peroxisomes ${ }^{26}$, in Xenopus extracts (Supplementary Fig. 5g). It acted similarly to the APH of the 
a

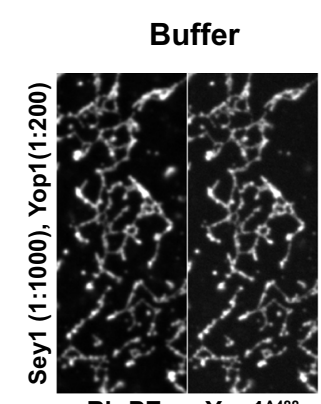

Rh-PE Yop1 ${ }^{\text {A488 }}$

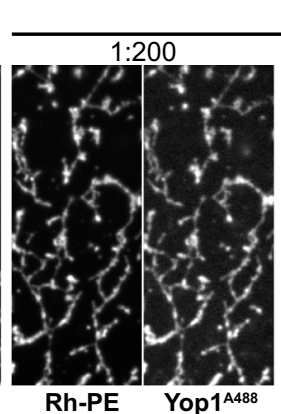

C

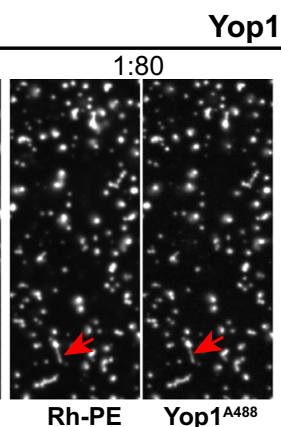

Rh-PE
Yop1 APH

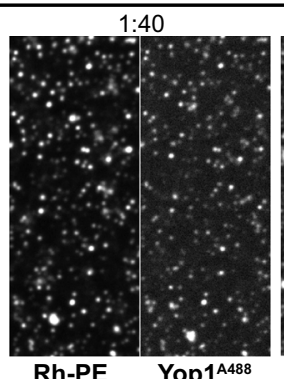

d

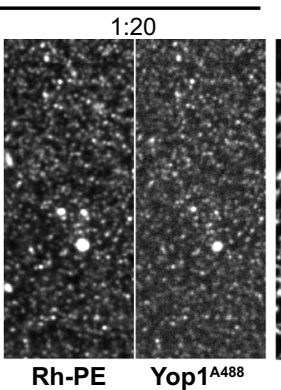

Yop1

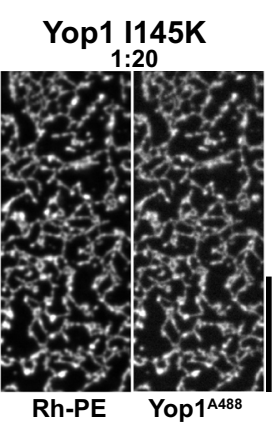

Rh-PE Yop1 ${ }^{\text {A488 }}$

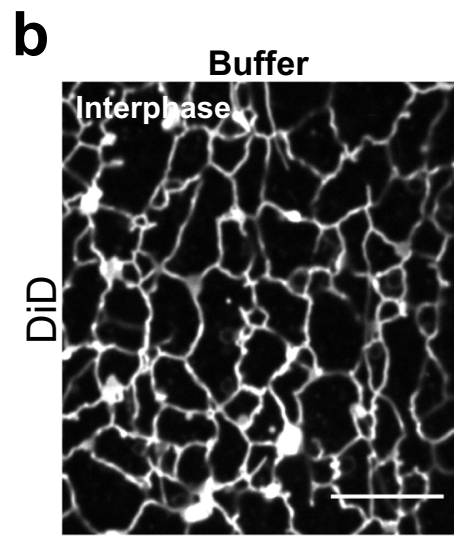

$\mathbf{f}$

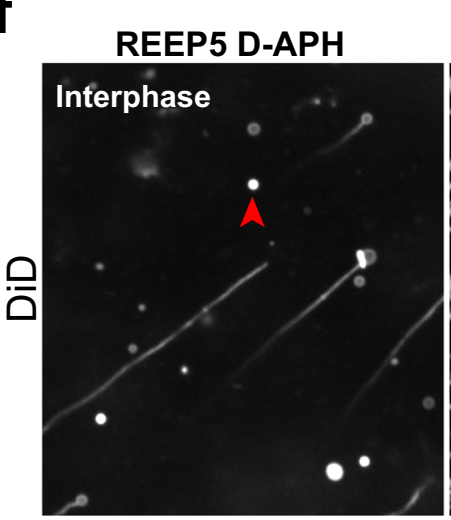

e
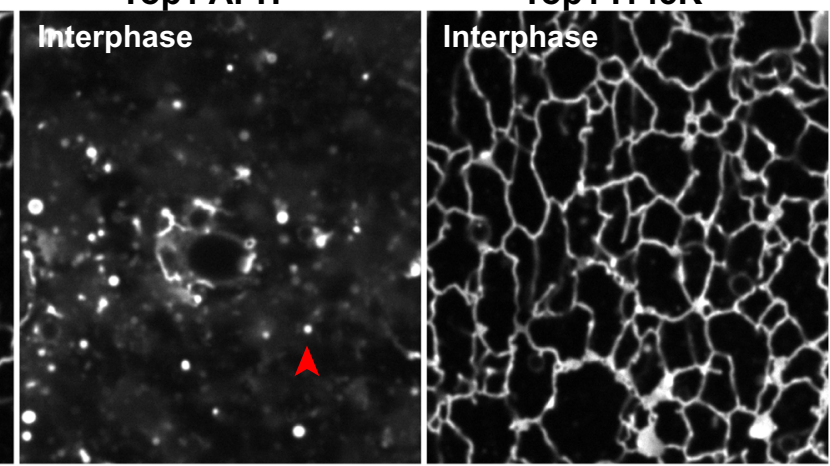

REEP5 L-APH Interphase

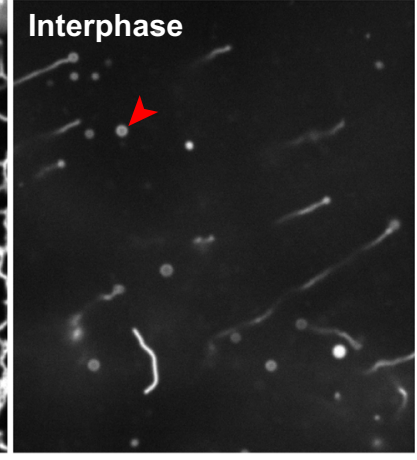

h

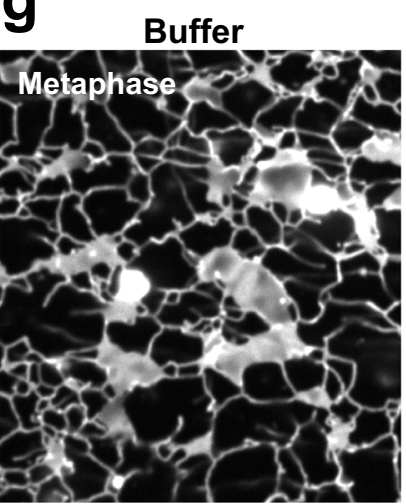

REEP5 L-APH

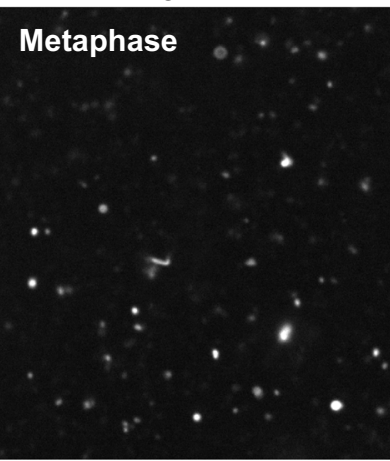

REEP5 D-APH

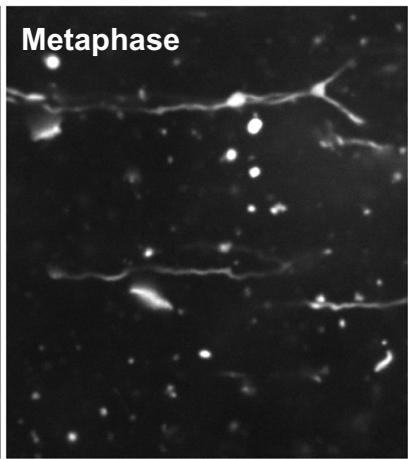

Fig. 7 APH peptides abolish membrane networks in a reconstituted system and Xenopus egg extracts. a Alexa 488-labeled Yop1 (Yop1A488) was coreconstituted with the GTPase Sey1 into liposomes containing S. cerevisiae lipids and fluorescent Rh-PE at the protein to lipid ratios given on the left. Synthetic peptides corresponding to the APH of wild-type Yop1 or to a mutant in the APH (Yop1 I145K) were added at the molar ratios (peptide to lipid)

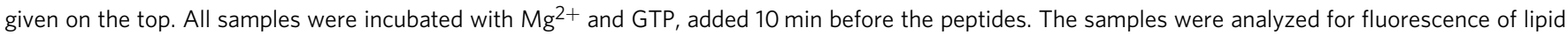
and protein. Arrows point to residual short tubules. The experiment was performed two times. b An interphase tubular network was generated with Xenopus egg extract and stained with the fluorescent dye DiD. The network was visualized with a fluorescence microscope with excitation at 642 nm. Bar, $10 \mu \mathrm{m}$. c As in $\mathbf{b}$, but in the presence of $100 \mu \mathrm{M}$ of a synthetic peptide corresponding to wild-type Yop1 (Yop1 APH). d As in $\mathbf{b}$, but with a peptide carrying a point mutation in the APH (Yop1 1145K). e As in b, but with a peptide corresponding to the APH of wild-type Xenopus REEP5 (REEP5 L-APH). $\mathbf{f}$ As in e, but with a peptide containing D-amino acids (REEP5 D-APH). These experiments were performed four times. $\mathbf{g}$ Network generated with Xenopus egg extract in metaphase. $\mathbf{h}$ As in $\mathbf{g}$, but in the presence of REEP5 L-APH. i As in $\mathbf{g}$, but in the presence of REEP5 D-APH. These experiments were performed three times.

REEPs, again converting tubules into small membrane fragments. In contrast, a mutant peptide reported to be inactive in peroxisome tubulation (referred to as Pex11 3E because it carries Glu mutations at positions I69, I72, and F75 ${ }^{26}$ ) was inactive (Supplementary Fig. 5g). Interestingly, the APH of Sey1 had no effect on the tubular network in Xenopus extracts (Supplementary Fig. 5g). In contrast to the APHs of the REEPs, this APH may not penetrate the membrane to an optimal depth to cause sufficient change in the monolayer spontaneous curvature $^{15}$.
The APH is required for Rtn's exclusive ER-tubule localization and restricted mobility. To test the role of the APH in vivo, we used the fission yeast $S$. japonicus, which has large cells that facilitate live-cell imaging ${ }^{27}$. S. japonicus has one member each of the Rtn and REEP protein families (Rtn1 and Yop1, respectively). We focused on Rtn 1 because this protein localizes exclusively to the cortical tubular ER network and is absent from the nuclear envelope when expressed at endogenous levels (Fig. 8a); in contrast, Yop1 localizes to both the cortical ER and nuclear pores (Supplementary Fig. 6a, b), which complicates the analysis. 


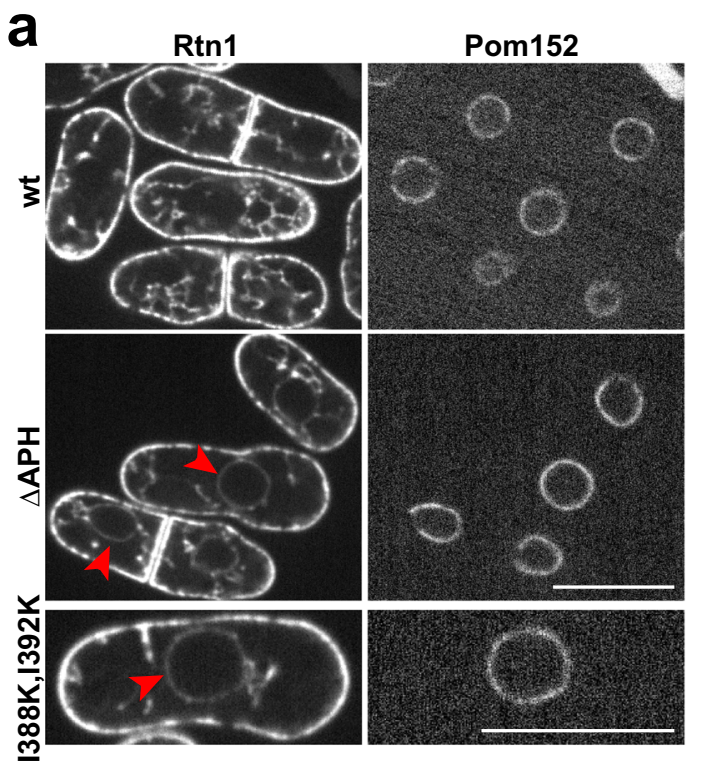

b

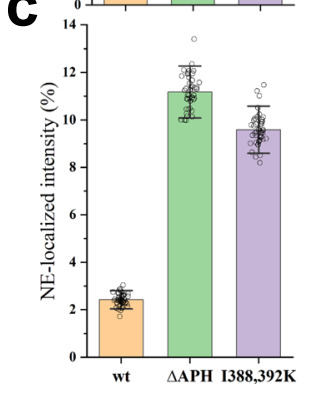

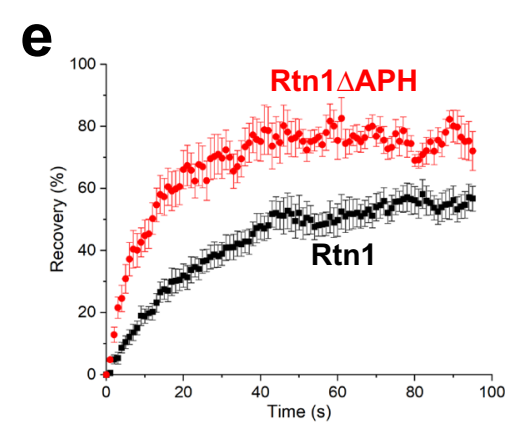

f

\begin{tabular}{c|c} 
Protein & Recovery half time (s) \\
\hline Rtn1 & $16.8 \pm 2.3$ \\
Rtn1 $\Delta$ APH & $7.4 \pm 1.5$ \\
Yop1 & $11.5 \pm 2.0$ \\
Yop1(I145K) & $5.6 \pm 1.2$ \\
\hline
\end{tabular}
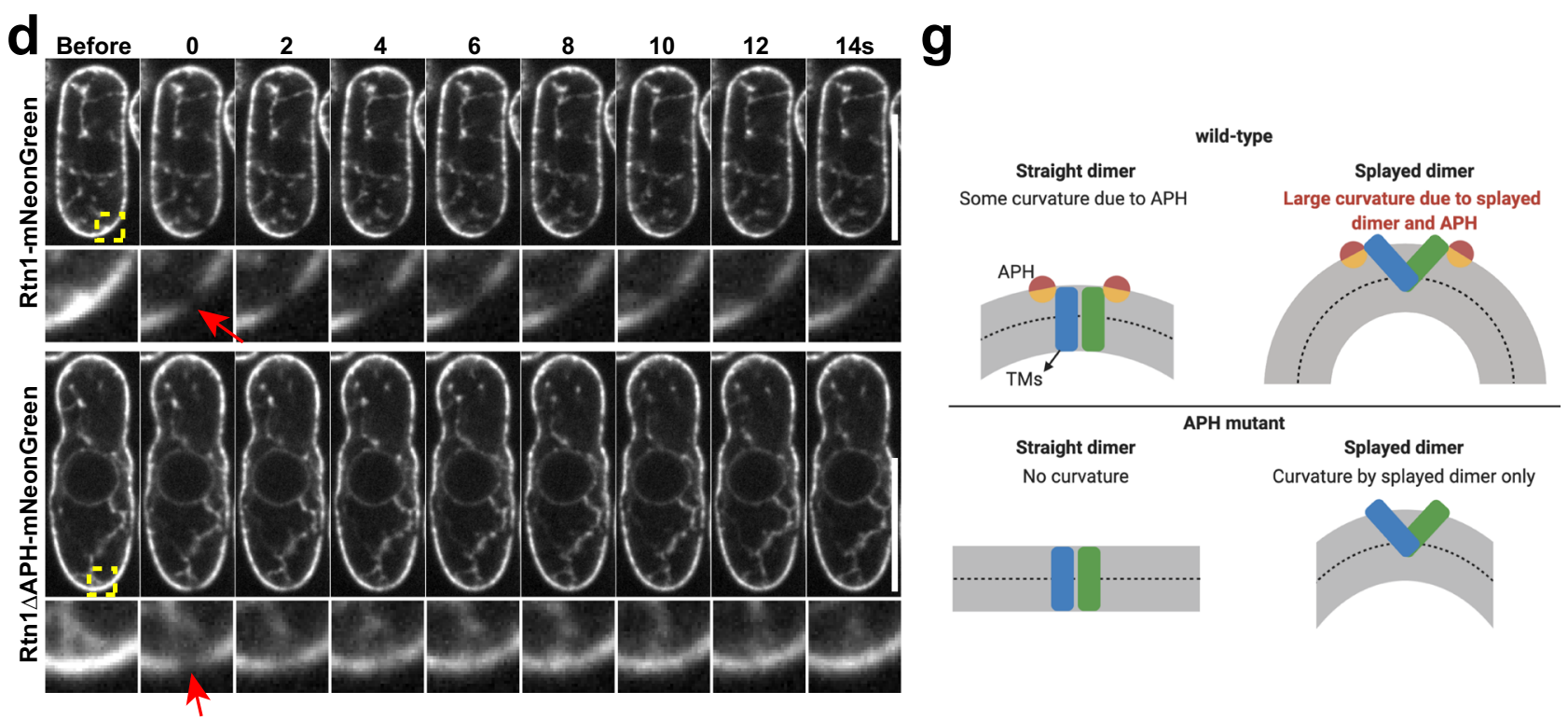

Fig. 8 The APH is required for Rtn1's exclusive ER-tubule localization and restricted mobility in S. japonicus cells. a Fusions of mNeonGreen with wildtype Rtn1 or APH mutants ( $\triangle \mathrm{APH}$, and I388K, I392K) were expressed in S. japonicus cells under rtn1's native promoter. The cells also expressed a mCherryfusion of Pom152 as a marker for nuclear pores and the nuclear envelope (NE). The cells were visualized with a confocal fluorescence microscope. Shown are representative images focused on the center of the cells obtained from three independent experiments. Arrowheads point to NE-localized mutant Rtn1. b Quantification of total fluorescence intensity. Background fluorescence acquired from untagged cells was subtracted. The total fluorescence of the mutants was normalized to that of wild-type cells. c Quantification of NE-localized fluorescence intensity. The Rtn1 fluorescence co-localizing with Pom 152 was determined, the cytoplasmic background was subtracted, and the difference divided by the total intensity. Data are presented as mean \pm SD of $n=49$ (wt), $43(\Delta \mathrm{APH})$, and 46 (I388K, I392K) cells for each strain from two independent experiments. d Cells expressing Rtn1-mNeonGreen or Rtn1 $\Delta$ APHmNeonGreen were bleached at their periphery and the recovery of fluorescence followed over time (FRAP). The box shows the bleached area. The lower panels show magnified views. The arrows point to the bleached region of the peripheral ER. Bars, $10 \mu \mathrm{m}$. e Quantification of FRAP experiments. Fluorescence intensity before and immediately after bleaching was set to $100 \%$ and $0 \%$, respectively. Error bars are standard error of the mean (SEM) of each time point from nine Rtn1-mNeonGreen-expressing cells and ten Rtn1 $\Delta$ APH-mNeonGreen-expressing cells. f Recovery half-times ( \pm SEM) were calculated from FRAP experiments, such as shown in d. The analysis for Rtn1, Rtn1 $\Delta$ APH, Yop1, and Yop1 (I145K) is based on 9, 10, 14, 11 cells from three independent experiments, respectively. $\mathbf{g}$ Model for membrane curvature generation by REEP dimers. The TMs of REEPs can form either straight dimers (monomers represented by green and blue rods) that span the lipid bilayer (gray) or splayed dimers that sit predominantly in the outer leaflet of the bilayer. The APH (red/orange circle) promotes the formation of splayed dimers by generating local curvature through hydrophobic insertion. APH mutants can still generate membrane curvature when their local concentration is sufficiently high. 
When the APH of Rtn1 was deleted, or when charges were introduced into the hydrophobic face of the helix, the protein was seen both in the cortical tubular ER network and the nuclear envelope (Fig. 8a; quantification in Fig. 8b, c). The Rtn1 mutant proteins localized to the low-curvature membranes of the nuclear envelope, rather than to the curved membranes around the nuclear pores (see scheme in Supplementary Fig. 6c): the deletion of the nucleoporin Nup132, caused pore clustering ${ }^{28,29}$, but only small changes in the localization of the Rtn1 mutants (Supplementary Fig. 6d). These results, therefore, show that the APH is required for the exclusive localization of Rtn1 to the tubular ER, i.e., the region of high membrane curvature.

When the APH of Rtn1 was replaced by that of Yop1, the protein mostly localized to the cortical ER (Supplementary Fig. 6e; quantification in Supplementary Fig. 6f), indicating that it is partially functional. Similar effects were seen with the APH of Pex11, while the 3E mutant was inactive (Supplementary Fig. 6e, f). The amphipathic helix ALPS of the ArfGAP1 protein ${ }^{30}$ was also inactive (Supplementary Fig. 6e, f); again, in contrast to the APHs of the reticulons and REEPs, this APH may not penetrate the membrane monolayer to an optimal depth.

Finally, we tested whether the APH affects the diffusional mobility of Rtn 1 in the plane of the membrane. To this end, we expressed Rtn1 from the chromosome under its endogenous promoter as a fusion with the fluorescent protein $\mathrm{mNeonGreen}$ and performed Fluorescence Recovery After Photobleaching (FRAP) experiments on live cells. Upon bleaching of a small area, the recovery of fluorescence was followed over time (Fig. 8d). The recovery with a wild-type Rtn 1 fusion was relatively slow, consistent with previous reports ${ }^{14,21}$. The recovery was considerably faster when the APH of Rtn1 was deleted (Fig. 8d; quantification in Fig. 8e, f). A fusion of Yop1 with mNeonGreen also diffused only slowly in the membrane, while the I145K mutant moved considerably faster (Fig. 8f). Thus, the APH seems to affect the mobility of both Rtn1 and Yop1, perhaps by promoting the formation of larger assemblies.

\section{Discussion}

Here, we have analyzed the molecular mechanism by which the REEPs generate the high membrane curvature that is characteristic of ER tubules in cross-section. Our results show that the basic unit of the REEPs are dimers, rather than monomers, as previously believed. The two monomers interact within the membrane, with TM2 at the interface. Both features of the REEPs, the TMs, and the APH, contribute to generating high membrane curvature. The TMs alone can generate extreme curvature when present at high concentrations, converting phospholipid bilayers into micelle-like lipoprotein particles (LPPs). The APH is also capable of producing high membrane curvature, converting tubules into small vesicles or LPPs. As discussed below, these results have implications for how ER tubules are formed. Our conclusions are probably applicable to the Rtns as well because these proteins also have two hairpin TMs and an APH, they can replace the REEPs in forming an ER network, and Rtn1's APH is important for its function and can be swapped with that of Yop1. Nevertheless, it remains to be tested whether Rtns form stable dimers and can generate LPPs. The ER-phagy receptor FAM134B also has a similar structure as the REEPs and Rtns, and molecular dynamics simulations of a membrane-embedded monomer show that again both the TMs and the APH are required for curvature generation $^{31}$. Although FAM134B formed clusters that locally bent the bilayer, no LPP formation was observed, perhaps because dimerization through the TMs was not considered.

The most surprising finding of our study is that, at high concentrations, the REEPs can generate so much curvature that a lipid bilayer is converted into a micelle-like lipoprotein particle (LPP). To our knowledge, the REEPs are the first transmembrane proteins that can form LPPs. NMR studies suggested that both TM hairpins span a phospholipid bilayer ${ }^{13}$, but there are only a few hydrophilic or charged residues at the tips of the hairpins and the REEPs can therefore sit both in a bilayer with an aqueous lumen and a monolayer of a micelle lacking a lumen. Other known LPP-forming proteins, such as high-density lipoprotein, contain short 11 amino acid repeats that form amphipathic helices 32,33 . The apoprotein wraps around the lipid core, with the hydrophobic sides of the helices facing the lipids. A similar structure has been suggested for a-synuclein, which also has multiple amphipathic helical segments, but no TM domains ${ }^{34,35}$. a-synuclein can form LPPs of similar dimensions as those generated by the REEPs, and like the REEPs, can generate tubules and small vesicles from larger vesicles ${ }^{34,35}$. However, the cellular function of $a$-synuclein remains obscure. Our in vitro experiments suggest that the REEPs may form LPPs by budding from larger vesicles, a process that would be analogous to the formation of micelles by detergent addition to liposomes ${ }^{36}$.

Previous models postulated that the REEPs and Rtns generate membrane tubules not only by hydrophobic insertions but also by forming arc-like scaffolds around tubules ${ }^{6}$. This model was largely based on the mechanism of BAR-domain proteins, which are cytosolic proteins forming crescent-shaped, rigid dimers that attach to the cytoplasmic face of a membrane and impose their curvature on that of the lipid bilayer ${ }^{37,38}$. This scaffolding mechanism requires that the bending rigidity of the oligomers is larger than that of the membrane and that the binding energy between the monomers is sufficiently strong. The REEP/Rtn dimers might form oligomeric assemblies, as they diffuse only slowly in the plane of the membrane and generate a ladder in cross-linking experiments ${ }^{13,21}$. Our FRAP experiments also suggest that the APH promotes the association of dimers, although the APH probably acts indirectly through altering local membrane stress, because it does not crosslink to any parts of the protein. However, even if the REEPs/Rtns formed oligomers, it seems unlikely that these would be rigid structures that could mold a lipid bilayer similarly to the BAR-domain proteins, as they lack folded cytosolic segments and would have to form a scaffold from the TM segments.

Although the TMs likely do not form a rigid scaffold, they play a major role in generating high membrane curvature, as Yop1 lacking its APH can still generate LPPs at high protein concentrations. The mechanism by which the TMs generate curvature likely relates to how they are accommodated in a lipid monolayer or bilayer. If the TMs were oriented perpendicular to the membrane surface, they would produce no or little crossmembrane asymmetry and thus no substantial membrane curvature (Fig. 8g; straight dimers). We, therefore, propose that a REEP dimer has the propensity to have a splayed conformation in which the two monomers, and therefore their TMs, are tilted relative to one another (Fig. 8g; splayed dimers). The splayed dimer would sit mostly in the cytoplasmic monolayer of the ER membrane and form a strongly asymmetric structure across the monolayer. Such a structure would generate monolayer spontaneous curvature that promotes bending of the cytoplasmic monolayer. Because of the trans-monolayer coupling in a lipid bilayer, the whole-membrane curves, while the luminal monolayer resists this bending. As a result, both monolayers accumulate elastic stress. At high protein concentrations, the spontaneous curvature of the cytoplasmic monolayer becomes so large that the resulting elastic stresses disrupt the bilayer, converting it into the highly curved monolayer of an LPP. This mechanism is analogous to that established for the curvature stress-induced conversion of bilayers into micelles during membrane solubilization with 
common detergents ${ }^{39,40}$. A collective transition of non-splayed to splayed dimers would be facilitated by their dense packing, as there would be little lipids left that could resist membrane bending. Our results show that REEPs from different species can generate different spontaneous curvature, which may be explained by slight variations in the tilting of monomers within the dimer. Perhaps, this causes the diameter of ER tubules to differ among organisms. Our model of curvature generation by splayed dimers is different from the previously considered mechanisms of hydrophobic APH insertion and scaffolding.

One mechanism by which the APH generates high membrane curvature is hydrophobic insertion. Our experiments with synthetic peptides show that the addition of APH is sufficient to break up tubules or large liposomes into small vesicles or LPPs. Because APH peptides of different chirality have the same effect, the helix directly deforms the lipid bilayer, rather than acting indirectly on other parts of the protein. The APH likely shallowly inserts into the outer leaflet of the lipid bilayer, displacing the polar head groups of the surrounding phospholipid molecules, and causing this monolayer to locally acquire a large positive spontaneous curvature. The hydrophobic insertion mechanism of the APHs ${ }^{15}$ has been proposed for REEPs and Rtns and was originally shown for epsin and N-BAR-domain containing proteins $^{37,41,42}$.

Our cross-linking results suggest that the APH also affects the relative orientation of the monomers in the dimer, suggesting that the APH not only acts through hydrophobic insertion but also by favoring a curvature-generating splayed dimer conformation. This is likely not a direct effect of the APH on the protein conformation, because no photo-cross-links were seen with any of the residues of the APH. Rather, the APH may affect the relative position and orientation of the REEP monomers indirectly by facilitating local membrane bending and thus promoting the splayed dimer conformation. In the absence of the APH, there would be a mixture of splayed and straight dimers. At high concentrations, APH-lacking molecules would still form splayed dimers through their TMs and thus generate LPPs (Fig. 8g). Interconversion between straight and splayed dimers is supported by the different cross-linking yields seen with REEPs localized to the monolayer of LPPs or the bilayer of membranes. Thus, we propose that the APH increases the local membrane monolayer spontaneous curvature both directly by hydrophobic insertion, and indirectly by promoting the transition of straight to splayed dimers.

The fusion GTPase ATL can also generate high membrane curvature, similarly to the REEPs and Rtns, as it alone is sufficient to form a GTP-dependent tubular membrane network in a reconstituted system ${ }^{11}$. Interestingly, ATL seems to have the same membrane topology as the REEPs and Rtns ${ }^{43}$, and it forms dimers even in its GDP-bound state ${ }^{44-46}$, likely by interactions of the TMs inside the membrane ${ }^{47}$. In the GDP-bound state, ATL causes the breakup of tubules into small vesicles or membrane fragments ${ }^{12}$, indicating that its GTPase-dependent fusion activity counteracts the excessive curvature generated by the TMs. The $\mathrm{APH}$ of ATL also interacts with lipids, which stimulates fusion $^{47,48}$, but it does not disrupt the ER network in Xenopus extracts. Nevertheless, the similarities between ATL and the REEPs and Rtns suggests that all these proteins use splayed dimers to generate high membrane curvature.

In summary, we propose a model (Fig. 8g) by which REEPs generate high membrane curvature by two mechanisms, the formation of splayed dimers by the TMs, structures that are asymmetric across the membrane, and the hydrophobic insertion of the $\mathrm{APH}$ in the cytoplasmic leaflet of the bilayer. The splayed dimer and the APH each generate local monolayer spontaneous curvature, which leads to the local bending of the membrane. Ensembles of such domains cause the formation of either cylindrical tubules or spherical vesicles. Under physiological conditions, tubules would be favored over vesicles. Tubules are favored kinetically, as the transition from an initially flat membrane into a cylindrical tubule does not require membrane fission, in contrast to the formation of a vesicle. Tubules could also be favored thermodynamically if the projection of the dimers onto the membrane plane has a noncircular shape. Such a lateral anisotropy would favor anisotropic over the isotropic realization of the spontaneous mean curvature. Tubules would also be favored over vesicles if the dimers interacted even weakly to form elongated assemblies. Factors promoting the breakup of tubules into vesicles include the rotational entropy of the dimers, which would counteract lateral anisotropy of the dimers, and a negative modulus of the Gaussian curvature predicted from the generation of positive spontaneous curvature in the cytoplasmic membrane leaflet, which would favor tubule fission into spheres ${ }^{38}$. Regardless of whether tubules are favored over vesicles kinetically or thermodynamically, our model predicts that the REEPs form large vesicles at low concentrations, tubules at intermediate concentrations, and small vesicles and eventually LPPs at high concentrations. At physiological concentrations, tubules would predominate. The proposed model is not only consistent with the in vitro data presented, but also with the fact that overexpression of an Rtn leads to long, narrow, and stiff tubules, which tend to break up into smaller fragments $^{12,21}$. Our model does not require the dimers to form higher oligomers, but oligomerization would counteract entropic effects and could increase the curvature anisotropy, which would help to segregate REEPs and Rtns in tubules and sheet edges, despite them being able to diffuse into flat sheets.

\section{Methods}

Protein expression and purification (membrane fraction). The codonoptimized gene for $S$. japonicus Yop1 was cloned into a modified pET21b vector that encoded a C-terminal 3C protease site followed by an SBP tag, as previously described ${ }^{11}$. A modified $\mathrm{pET} 28 \mathrm{~b}$ vector coding for a C-terminal TEV protease site followed by an SBP tag was used for the expression of $X$. laevis REEP5. Sitedirected mutagenesis was used to generate mutants of Yop1 and REEP5. The sequence of BRIL was synthesized as a gene block and inserted at the desired position of Yop1 or REEP5 using Gibson assembly. Plasmids and primers used in this study are listed in Supplementary Tables 1 and 2.

Yop1 and REEP5 were expressed and purified as SBP fusion proteins in E. coli BL21-CodonPlus (DE3)-RIPL (Agilent). Cells were cultured in 2x YT media, and expression was induced at $\mathrm{OD}_{600} \sim 0.6$ with $250-400 \mu \mathrm{M}$ isopropyl- $\beta$-D-

thiogalactopyranoside (IPTG) at $16^{\circ} \mathrm{C}$ for $16-18 \mathrm{~h}$. The cell pellet was washed once and sonicated in lysis buffer containing $25 \mathrm{mM}$ HEPES/KOH pH 7.4, $150 \mathrm{mM}$

$\mathrm{NaCl}$, and protease inhibitors. The lysate was cleared with low-speed centrifugation and centrifuged at $44,000 \times g$ for $1 \mathrm{~h}$ to sediment membranes. The membranes were solubilized for $1 \mathrm{~h}$ with $1 \% \mathrm{n}$-dodecyl- $\beta$-maltoside (DDM) at $4{ }^{\circ} \mathrm{C}$. Insoluble material was removed by centrifugation at $50,000 \times \mathrm{g}$ for $30 \mathrm{~min}$. The clarified supernatant was incubated with streptavidin agarose resin for $1 \mathrm{~h}$ at $4{ }^{\circ} \mathrm{C}$. The resin was washed with 10-15 volumes of lysis buffer containing $0.03 \%$ DDM. Protease inhibitors were left out for the last five washing steps. Proteins were eluted by oncolumn cleavage of SBP with 3C (Yop1) or TEV (REEP5) protease overnight at $4^{\circ}$ $\mathrm{C}$ in a buffer containing $25 \mathrm{mM}$ HEPES/KOH pH 7.4, $100 \mathrm{mM} \mathrm{NaCl}, 0.03 \%$ DDM. In all experiments, $2 \mathrm{mM}$ dithiothreitol (DTT) was added for the purification of $X$. laevis REEP5. Purification of S. cerevisiae Sey1 was as described in ref. ${ }^{11}$. All membrane proteins were further purified by SEC on a Superdex 200 or Superose 6 column (GE Healthcare) and concentrated by ultrafiltration (Amicon Ultra, EMD Millipore).

To incorporate Bpa probes into REEP/Yop1, the mutated REEP expression plasmid and the amber suppressor tRNA plasmid were co-expressed in BL21 (DE3) cells $(\mathrm{NEB})^{49}$. The expression steps were similar to those with wild-type Yop1, except that $1 \mathrm{mM}$ final concentration of $\mathrm{H}-\mathrm{Bpa}-\mathrm{OH}$ (Chempep) was added and amber suppressor was induced 1-2 $\mathrm{h}$ before IPTG addition.

Purified Yop1 in DDM was labeled with Alexa Fluor NHS ester dyes (Thermo Fischer Scientific) as described in ref. ${ }^{11}$. The labeling efficiency was calculated by comparing the absorbance of the protein at $280 \mathrm{~nm}$ and the absorbance of the dye at $556 \mathrm{~nm}$ for Alexa Fluor 546 and $495 \mathrm{~nm}$ for Alexa Fluor 488, using a NanoDrop 2000c Spectrophotometer (Thermo Scientific).

Multiangle light scattering (MALS). Affinity-purified REEP5 or Yop1 was subjected to gel filtration on a Superdex 200 column in a buffer containing $20 \mathrm{mM}$ Tris/ $\mathrm{HCl} \mathrm{pH} 7.5,100 \mathrm{mM} \mathrm{NaCl}$, and $0.03 \%$ DDM. Approximately $100 \mu$ l fractions 
of the peak were collected (protein concentration $0.2-1 \mathrm{mg} / \mathrm{ml}$ ) and used for SECMALS. Protein samples were passed through an Agilent Advance Bio 300 column on an Agilent liquid chromatography system, a DAWN HELEOS II multiangle light scattering detector, an Optilab T-rEX refractive index detector, and UV detector (Wyatt Technology Corporation). Data were processed in ASTRA 6 using the protein conjugate model. We used $\mathrm{d} n / \mathrm{d} c$ values of 0.185 for proteins and 0.133 for $\mathrm{DDM}^{50}$

Purification of Fabs directed to BRIL. Generation and cloning of Fab fragments against the BRIL fusion protein were described in ref. ${ }^{18}$. Fab fragments were expressed in E. coli BL21-CodonPlus (DE3)-RIPL. Cells were cultured in 2x YT media at $37^{\circ} \mathrm{C}$, and the expression was induced with $1 \mathrm{mM} \mathrm{IPTG}$ for $4 \mathrm{~h}$ at $37^{\circ} \mathrm{C}$ when the OD600 reached $0.6-0.8$. Cells were lysed by sonication, and the lysate was cleared by centrifugation at $50,000 \times g$ for $30 \mathrm{~min}$. The supernatant was passed over 1-2 $\mathrm{ml}$ of Protein A Sepharose $4 \mathrm{~B}$ resin (ThermoFisher), pre-equilibrated in 50 $\mathrm{mM}$ HEPES/KOH pH 7.5, $300 \mathrm{mM} \mathrm{NaCl}$. The resin was washed with 10 column volumes of buffer. Fabs were eluted with $0.1 \mathrm{M}$ acetic acid, and fractions were collected dropwise into $1.5-\mathrm{ml}$ tubes containing buffer to bring the final concentration in each tube to $100 \mathrm{mM}$ Tris/ $\mathrm{HCl} \mathrm{pH} 8.0,100 \mathrm{mM} \mathrm{NaCl}$. Fractions containing Fabs were combined, concentrated on Amicon $30 \mathrm{kDa}$ molecular weight cut-off filters, and further purified by SEC on a Superdex 200 column equilibrated in $20 \mathrm{mM}$ HEPES/KOH pH 7.5, $150 \mathrm{mM} \mathrm{NaCl}$. All purification steps were carried out at $4{ }^{\circ} \mathrm{C}$.

To confirm Yop1 is a dimer in detergent, SEC-purified Yop1-BRIL or Yop1 (I145R)-BRIL in DDM were incubated with a 2.5 -fold molar excess of BRIL Fabs for $1 \mathrm{~h}$ at $4{ }^{\circ} \mathrm{C}$, followed by subsequent chromatography on a Superose 6 column equilibrated in $25 \mathrm{mM}$ HEPES/KOH pH 7.4, $100 \mathrm{mM} \mathrm{NaCl}, 0.03 \%$ DDM. The peak fractions were collected and diluted to $10 \mu \mathrm{g} / \mathrm{ml}$ for negative-stain EM analysis.

Reconstitution of REEPs. All lipids were obtained as chloroform stocks from Avanti Polar Lipids. Liposomes made of S. cerevisiae Yeast Polar Lipid Extract were prepared as previously described in ref. ${ }^{11}$ with minor changes. The chloroform lipid mixtures were first dried under a nitrogen stream and then under vacuum overnight. Dried lipid films were hydrated in buffer containing $25 \mathrm{mM}$ HEPES/ $\mathrm{KOH} \mathrm{pH} 7.4,100 \mathrm{mM} \mathrm{NaCl}, 1 \mathrm{mM}$ EDTA, and 5\% glycerol and subjected to ten freeze-thaw cycles.

Detergent-mediated reconstitution was used to integrate all proteins into liposomes ${ }^{11}$. Briefly, liposomes and protein were incubated together at the desired protein:lipid ratio and supplemented with DDM to reach an estimated final concentration of $\sim 0.1 \%$. The mixture was incubated with gentle rotation at $4{ }^{\circ} \mathrm{C}$ for $1 \mathrm{~h}$. The detergent was then removed by four successive additions of Bio-Beads SM2 Resin (Bio-Rad) over the course of $\sim 24 \mathrm{~h}$ at $4{ }^{\circ} \mathrm{C}$ or $4 \mathrm{~h}$ at room temperature. Insoluble aggregates were removed by centrifugation.

Nycodenz gradient centrifugations were performed as follows: $25 \mu$ of the reconstituted sample or peptide-bound liposomes were mixed with $25 \mu \mathrm{l}$ of $80 \%$ $(\mathrm{w} / \mathrm{v})$ Nycodenz and placed at the bottom of a Beckman polypropylene $5 \times 20-\mathrm{mm}$ tube. In total, $50 \mu \mathrm{l}$ of $30 \%, 20 \%, 10 \%$, and $0 \%$ Nycodenz were added sequentially on top. Samples were centrifuged at 50,000 rpm $(\sim 225,000 \times g)$ for $1 \mathrm{hr}$ in Optima TLX ultracentrifuge using a TLS55 rotor. Five $50 \mu \mathrm{l}$ fractions were collected from the top and analyzed by SDS-PAGE.

Photo-cross-linking experiments. Purified Bpa incorporated REEP/Yop1 mutants $(5 \mu \mathrm{M})$ in buffer containing $0.03 \%$ DDM or reconstituted into liposomes generated with a S. cerevisiae lipid extract at a protein:lipid molar ratio of 1:200 were added to Thermowell PCR tubes (Corning) and placed on an ice-cold metal block. A longwave UV lamp (Blak-Ray) was positioned $5 \mathrm{~cm}$ from the tubes, and the samples were irradiated for $30 \mathrm{~min}$. After irradiation, SDS sample buffer was added. The samples were then subjected to SDS-PAGE and Coomassie-blue staining.

Purification of REEP5 lipoprotein particles (LPPs) from E. coli. To purify $X$. laevis REEP5 LPPs from E. coli, the supernatant of the $44,000 \times g$ centrifugation step was collected and incubated with streptavidin agarose resin with gentle rotation for $1 \mathrm{hr}$ at $4{ }^{\circ} \mathrm{C}$. The resin was washed extensively with 15 resin volumes of buffer containing $25 \mathrm{mM}$ HEPES/KOH pH 7.4, $300 \mathrm{mM} \mathrm{NaCl}, 5 \mathrm{mM} \mathrm{MgCl}, 0.1 \%$ $(\mathrm{w} / \mathrm{v})$ ATP, $2 \mathrm{mM}$ DTT, and protease inhibitors, then with 15 resin volumes of the buffer without protease inhibitors. LPPs were eluted by on-column cleavage of the SBP tag with TEV protease overnight at $4{ }^{\circ} \mathrm{C}$. The eluted material was further purified by SEC on a Superose 6 column with lysis buffer supplemented with $2 \mathrm{mM}$ DTT and 5\% glycerol.

To determine the relative distribution of REEP5 in the sedimentable membrane and non-sedimentable supernatant fractions, SDS sample buffer was added to equivalent amounts of total lysate, membranes (resuspended in the same volume as the supernatant), or supernatant. The samples were heated at $65^{\circ} \mathrm{C}$ for $10 \mathrm{~min}$ and then subjected to SDS-PAGE. Proteins were transferred to PVDF membranes with a Bio-Rad Turbo transfer system and probed with DyLight 800-labeled streptavidin (Invitrogen) diluted 1:2000 in 1\% BSA. The membranes were scanned with an Odyssey imager (LI-COR).

To analyze the Fab-bound REEP5-BRIL LPP complex, SEC-purified LPPs were incubated with a tenfold molar excess of BRIL Fabs for $1 \mathrm{hr}$ at $4^{\circ} \mathrm{C}$, followed by chromatography on a Superose 6 column equilibrated in $20 \mathrm{mM} \mathrm{HEPES/KOH} \mathrm{pH}$ $7.5,150 \mathrm{mM} \mathrm{NaCl}$. The peak fraction was collected and diluted to $5 \mu \mathrm{g} / \mathrm{ml}$ for negative-stain EM analysis.

Lipid extraction and TLC. Purified REEP5 LPPs containing $115 \mu \mathrm{g}$ of protein was extracted with the Bligh and Dyer Lipid Extraction method ${ }^{51}$, resulting in $50 \mu$ of the organic phase. 1-, 4-, and $10 \mu$ lof the organic phase was loaded on a silica plate (EMD Millipore) and run with $60 \%$ chloroform $/ 35 \%$ methanol/ $5 \% \mathrm{H}_{2} \mathrm{O}$ as a solvent. Lipids were stained with Primuline and visualized in a fluorescence scanner.

Reconstitution of a tubular network with purified Sey1 and REEPs. For reconstitution of a tubular membrane network, liposomes consisting of 65:18.5:15:1.5 mole percent of POPC:DOPE:DOPS: 1,2-dioleoyl-sn-glycero-3phosphoethanolamine-N-[lissamine rhodamine B sulfonyl] (Rh-PE), or S. cerevisiae extract liposomes, consisting of 98.5:1.5 mole percent Yeast Polar Lipid Extract:Rh-PE were used as previously described ${ }^{11}$. These liposomes were extruded 21 times through polycarbonate filters of $100-\mathrm{nm}$ pore size using a mini-extruder (Avanti Polar Lipids) at room temperature.

Co-reconstitution of Seyl and REEPs was essentially done as described in ref. ${ }^{11}$. The proteoliposome suspension was first supplemented with $5 \mathrm{mM} \mathrm{MgCl}_{2}$ Then, either $2 \mathrm{mM}$ GTP or an equal volume of buffer was added, and the reaction was incubated for $5-10 \mathrm{~min}$ in the dark at room temperature. The reactions were mounted between two PEGylated coverslips, sealed with Valap, and placed on a microscope stage for another 5-10 min to allow settlement of the network prior to imaging.

Network formation with REEP5-containing LPPs was similar, except that purified REEP5 LPPs were co-reconstituted with Sey1 into Rh-PE-labeled POPC: DOPE:DOPS liposomes at a 1:50 molar ratio of REEP5 to lipid in liposomes, and that the total concentration of DDM was $\sim 0.2 \%$ during the incubation.

Fluorescence microscopy. All fluorescence microscopy samples were visualized using a spinning-disk confocal mounted on a Ti-motorized inverted microscope (Nikon). Microscope settings were the same as previously described ${ }^{11}$. Green, red, and far-red fluorescence were excited with $488-\mathrm{nm}, 561-\mathrm{nm}$, and $642-\mathrm{nm}$ solidstate lasers. Images were acquired with a cooled CCD camera (Hamamatsu Photonics) controlled with MetaMorph and processed with Image J software.

Peptide synthesis and circular dichroism (CD) spectroscopy. Yop1 APH and I145K peptides were synthesized by the company Biosynthesis. Peptides corresponding to the APHs of Xenopus REEP5, Pex11, Pex11 3E, and of S. japonicus Seyl were synthesized by the company Genscript. The sequences of the peptides are listed in Supplementary Table 3. The peptides were dissolved in a buffer containing $25 \mathrm{mM}$ HEPES/KOH pH 7.4, $100 \mathrm{mM} \mathrm{KCl}$ at $2-4 \mathrm{mg} / \mathrm{ml}$. The exact concentration of the stock solutions was determined by adsorption at $280 \mathrm{~nm}$ using a Nano Drop 2000c spectrophotometer.

For CD analysis, the Yop1 and REEP5 APH peptides were diluted to $0.2 \mathrm{mg} / \mathrm{ml}$ with buffer containing $25 \mathrm{mM}$ Tris/ $\mathrm{HCl} \mathrm{pH}$ 8.0, $50 \mathrm{mM} \mathrm{NaCl}$. Liposomes containing a final concentration of $0.6 \mathrm{mg} / \mathrm{ml} \mathrm{S}$. cerevisiae lipids or $0.3 \% \mathrm{DDM}$ were added to test their effect on the secondary-structure formation of the peptides. The CD spectra were acquired on a Jasco J-815 spectropolarimeter equipped with a PFD-425S/15 Peltier unit. Spectra were accumulated 5 times from $250-200 \mathrm{~nm}$, using a scanning rate of $50 \mathrm{~nm} / \mathrm{min}$. Measurements were performed at $20^{\circ} \mathrm{C}$ in a 1 mm quartz cuvette.

ER network formation with Xenopus egg extracts. An interphase or metaphase ER network was formed using crude Xenopus egg extracts, as described previously $12,52,53$. The network was stained with $\mathrm{DiD}$ (1,1'-dioctadecyl-3,3,3', $3^{\prime}$ tetramethylindodicarbocyanine, 4-chlorobenzenesulfonate salt). The samples were mounted on PEGylated coverslips and visualized by a spinning-disk confocal microscope at a far-red channel. To test the effect of synthetic peptides, a final concentration of $100 \mu \mathrm{M}$ peptide was added to DiD-labeled interphase or metaphase extracts, and the samples incubated at room temperature for 5-10 min before imaging.

To determine the relative distribution of REEP5 in Xenopus egg extracts between a sedimentable membrane fraction and a non-sedimentable fraction, immunoblotting was performed with a polyclonal REEP5 antibody from Proteintech (used at 1:1000 dilution). Control blots were performed with calnexin antibodies (Enzo) (1:800 dilution) and with polyclonal antibodies raised against Xenopus Pex14 in rabbits, which were affinity-purified and used at a 1:10,000 dilution.

Negative-stain EM and Cryo-EM. For negative-stain EM analysis of reconstituted proteoliposomes or purified LPPs, $5 \mu$ of the sample was added to a glow-discharged carbon-coated copper grid (Pelco, Ted Pella Inc.) for 1 min. The excess sample was blotted off with filter paper. The grids were washed once with deionized water, and then stained once with freshly prepared $1 \%$ uranyl acetate for $30 \mathrm{~s}$ prior to blotting and air-drying. To determine the effect of peptides on naked liposomes by negative-stain EM, samples were applied to 
glow-discharged grids in a small petri dish with three drops of $2 \%$ osmium acid on the side without direct touching. Images were collected using a conventional transmission electron microscope Tecnai $\mathrm{G}^{2}$ Spirit BioTWIN operated at an acceleration voltage of $80 \mathrm{kV}$.

For negative-stain EM analysis of Fab-bound REEP5-BRIL LPPs and Fabbound Yop1-BRIL in DDM, $3.5 \mu \mathrm{l}$ of the sample was applied to grids for $45 \mathrm{~s}$. Excess sample was removed, and grids were immediately washed with deionized water, followed by two applications of freshly filtered $1.5 \%$ uranyl formate solution for a total of $45 \mathrm{~s}$ prior to blotting and air-drying. Grids were imaged by a ThermoScientific Tecnai T12 transmission electron microscope operating at 120 $\mathrm{kV}$ and equipped with a Gatan UltraScan $895 \mathrm{CCD}$ camera. Images were processed with RELION $3.0^{54}$.

For cryo-EM, sonicated S. cerevisiae extract liposomes $(2 \mathrm{mM})$ and purified REEP5 LPPs $(0.2 \mathrm{mg} / \mathrm{ml})$ were vitrified using a Vitrobot and Quantifoil R1/2 Copper grids. The grid was imaged in a FEI Tecnai F20 microscope operating at $200 \mathrm{KV}$. A total of 94 image stacks were acquired using a Gatan K2 Summit detector with a total dose of $50 \mathrm{e}-/ \AA 22$. Motion-corrected images are then processed and analyzed with RELION $3.0^{54}$

S. japonicus strain construction, cell culture, and imaging. Transformation of $S$. japonicus cells was performed as described ${ }^{55,56}$, with $\sim 10 \mu \mathrm{g}$ of purified DNA fragments per transformation. All transformants were verified by PCR. Supplementary Table 4 lists the S. japonicus strains used in this study. DNA fragments for gene targeting and gene deletion were designed as described ${ }^{56}$ with minor changes. Briefly, $\sim 500-1000$ bp homologous sequences flanking the designated insertion sites were used for homologous recombination. cDNA sequences carrying point mutations or short truncations were introduced into rtn1 or yop 1 knockout strains to generate tagged Rtn1 or Yop1 APH mutants. In most cases, genetic crossing was used to generate double or triple mutants. In the case of $r t n 1$ and pom152, the genetic loci were too close, so that a second round of transformation was needed.

The rich medium YE5S (yeast extract with five supplements) was used for $S$. japonicus cell culture. Cells were grown in YE5S for $\sim 24 \mathrm{~h}$ at $30^{\circ} \mathrm{C}$ before imaging, with proper dilutions to keep the culture in log phase. Live-cell imaging was performed in Bioptechs 0.17-mm Delta T TPG Dishes with a small piece of YE5S agar on top of the cells. A $z$-focal plane series was acquired at $0.2-0.4 \mu \mathrm{m}$ intervals spanning $8 \mu \mathrm{m}$ in most cases, but the middle focal plane is shown in the figures unless otherwise indicated.

The triple-knockout S. cerevisiae strain rtn1:Kan yop1:Kan nup85:HIS3 his3 ura3 leu2 harboring plasmid pSW3354 to express Nup85 was obtained from the laboratory of Susan Wente ${ }^{24}$. Yop1, Xenopus REEP5, S. japonicus Rtn1, and mutants were sub-cloned into the centromeric plasmid pRS415 under the control of S. cerevisiae Yopl's native promoter. This plasmid was transformed into yeast following the protocol described in ref. ${ }^{57}$. The cells were cultured in a synthetic medium lacking leucine and uracil. Serial fivefold dilutions were used to test growth on 5-fluoroorotic acid (5FOA) plates with an $\mathrm{OD}_{600}$ of 0.5 at the highest concentration. The final concentration of 5FOA was $1 \mathrm{mg} / \mathrm{ml}$.

Fluorescence recovery after photobleaching (FRAP). FRAP experiments with $S$. japonicus cells were conducted with a spinning-disk confocal microscope and a Photonic Instruments MicroPoint laser targeting system. Laser alignment was performed before each imaging session. Magnification, laser power, and detector gains were identical across all samples. The microscope was focused on the middle of the cells. Two to four cells were selected with a region of interest (ROI) of $\sim 10 \times$ 10 pixels for each FRAP experiment. Five pre-bleaching and 100 post-bleaching images were acquired with 1-s intervals. Photobleaching was performed at $100 \%$ laser power, and the selected region was bleached to $>50 \%$ of the original signal.

Data analysis was essential as described ${ }^{58}$. Briefly, after subtracting the background and correcting for the photobleaching during image acquisition, the ROI intensity was normalized with the mean pre-bleach intensity set to $100 \%$ and the intensity right after bleaching set at $0 \%$. Time 0 indicates the end time of bleaching. The equation $y=m_{1}+m_{2} \exp \left(-m_{3} x\right)$ was used to fit fluorescence recovery with Origin ( $\mathrm{m}_{3}$ is the off-rate). The half-time of recovery was calculated as $t_{1 / 2}=\ln 2 / \mathrm{m}_{3}$

Reporting summary. Further information on research design is available in the Nature Research Reporting Summary linked to this article.

\section{Data availability}

Data supporting the findings of this paper are available from the corresponding author upon reasonable request. A reporting summary for this article is available as a Supplementary Information file. Source data are provided with this link https://drive. google.com/file/d/liyqh68ZPwDM2cHQv7_7y46QdgHhMvREK/view?usp=sharing.

Received: 9 September 2020; Accepted: 7 December 2020;

Published online: 25 January 2021

\section{References}

1. Terasaki, M., Song, J., Wong, J. R., Weiss, M. J. \& Chen, L. B. Localization of endoplasmic reticulum in living and glutaraldehyde-fixed cells with fluorescent dyes. Cell 38, 101-108 (1984).

2. Shibata, Y., Voeltz, G. K. \& Rapoport, T. A. Rough sheets and smooth tubules. Cell 126, 435-439 (2006).

3. Shibata, Y. et al. Mechanisms determining the morphology of the peripheral ER. Cell 143, 774-788 (2010)

4. West, M., Zurek, N., Hoenger, A. \& Voeltz, G. K. A 3D analysis of yeast ER structure reveals how ER domains are organized by membrane curvature. $J$. Cell Biol. 193, 333-346 (2011).

5. Voeltz, G. K., Prinz, W. A., Shibata, Y., Rist, J. M. \& Rapoport, T. A. A class of membrane proteins shaping the tubular endoplasmic reticulum. Cell 124, 573-586 (2006).

6. Shibata, Y., Hu, J., Kozlov, M. M. \& Rapoport, T. A. Mechanisms shaping the membranes of cellular organelles. Annu. Rev. Cell Dev. Biol. 25, 329-354 (2009)

7. $\mathrm{Hu}$, J. et al. Membrane proteins of the endoplasmic reticulum induce highcurvature tubules. Science 319, 1247-1250 (2008).

8. $\mathrm{Hu}$, J. et al. A class of dynamin-like GTPases involved in the generation of the tubular ER network. Cell 138, 549-561 (2009).

9. Orso, G. et al. Homotypic fusion of ER membranes requires the dynamin-like GTPase Atlastin. Nature 460, 978-983 (2009).

10. Wang, N. \& Rapoport, T. A. Reconstituting the reticular ER networkmechanistic implications and open questions. J. Cell Sci. 132, (2019). https:// doi.org/10.1242/jcs.227611.

11. Powers, R. E., Wang, S., Liu, T. Y. \& Rapoport, T. A. Reconstitution of the tubular endoplasmic reticulum network with purified components. Nature 543, 257-260 (2017).

12. Wang, S., Tukachinsky, H., Romano, F. B. \& Rapoport, T. A. Cooperation of the ER-shaping proteins atlastin, lunapark, and reticulons to generate a tubular membrane network. eLife 5, e18605 (2016).

13. Brady, J. P., Claridge, J. K., Smith, P. G. \& Schnell, J. R. A conserved amphipathic helix is required for membrane tubule formation by Yop1p. Proc Natl Acad. Sci. USA 112, 639-648 (2015).

14. Breeze, E. et al. A C-terminal amphipathic helix is necessary for the in vivo tubule-shaping function of a plant reticulon. Proc. Natl Acad. Sci. USA 113, 10902-10907 (2016)

15. Campelo, F., McMahon, H. T. \& Kozlov, M. M. The hydrophobic insertion mechanism of membrane curvature generation by proteins. Biophys. J. 95, 2325-2339 (2008)

16. Zemel, A., Ben-Shaul, A. \& May, S. Modulation of the spontaneous curvature and bending rigidity of lipid membranes by interfacially adsorbed amphipathic peptides. J. Phys. Chem. B. 112, 6988-6996 (2008).

17. Chun, E. et al. Fusion partner toolchest for the stabilization and crystallization of G protein-coupled receptors. Structure 20, 967-976 (2012).

18. Mukherjee, S. et al. Synthetic antibodies against BRIL as universal fiducial marks for single-particle cryoEM structure determination of membrane proteins. Nat. Commun. 11, 1598 (2020).

19. Dormán, G. \& Prestwich, G. D. Benzophenone photophores in biochemistry. Biochemistry 33, 5661-5673 (1994).

20. Chin, J. W. et al. An expanded eukaryotic genetic code. Science 30, 964-967 (2003).

21. Shibata, Y. et al. The reticulon and Dp1/Yop1p proteins form immobile oligomers in the tubular endoplasmic reticulum. J. Biol. Chem. 283 , 18892-18904 (2008)

22. Rigaud, J. L., Pitard, B. \& Levy, D. Reconstitution of membrane proteins into liposomes: application to energy-transducing membrane proteins. Biochim Biophys. Acta 1231, 223-246 (1995)

23. Rigaud, J. L. \& Lévy, D. Reconstitution of membrane proteins into liposomes Methods Enzymol. 372, 65-86 (2003).

24. Dawson, T. R., Lazarus, M. D., Hetzer, M. W. \& Wente, S. R. ER membranebending proteins are necessary for de novo nuclear pore formation. J. Cell Biol. 184, 659-675 (2009).

25. Dreier, L. \& Rapoport, T. A. In vitro formation of the endoplasmic reticulum occurs independently of microtubules by a controlled fusion reaction. J. Cell Biol. 148, 883-898 (2000).

26. Opaliński, Ł., Kiel, J. A. K. W., Williams, C., Veenhuis, M. \& Van Der Klei, I. J. Membrane curvature during peroxisome fission requires Pex11. EMBO J. 30, 5-16 (2011).

27. Gu, Y., Yam, C. \& Oliferenko, S. Rewiring of cellular division site selection in evolution of fission yeasts. Curr. Biol. 25, 1187-1194 (2015).

28. Pemberton, L. F., Rout, M. P. \& Blobel, G. Disruption of the nucleoporin gene NUP133 results in clustering of nuclear pore complexes. Proc. Natl Acad. Sci. USA 92, 1187-1191 (1995).

29. $\mathrm{Li}, \mathrm{O}$. et al. Mutation or deletion of the Saccharomyces cerevisiae RAT3/ NUP133 gene causes temperature-dependent nuclear accumulation of poly(A) 
+ RNA and constitutive clustering of nuclear pore complexes. Mol. Biol. Cell 6, 401-417 (1995).

30. Bigay, J., Casella, J. F., Drin, G., Mesmin, B. \& Antonny, B. ArfGAP1 responds to membrane curvature through the folding of a lipid packing sensor motif. EMBO J. 24, 2244-2253 (2005).

31. Bhaskara, R. M. et al. Curvature induction and membrane remodeling by FAM134B reticulon homology domain assist selective ER-phagy. Nat. Commun. 10, 1-13 (2019).

32. Gogonea, V. Structural insights into high density lipoprotein: old models and new facts. Front. Pharmacol. 6, 318 (2016).

33. Gursky, O. Structural stability and functional remodeling of high-density lipoproteins. FEBS Lett. 589, 2627-2639 (2015).

34. Mizuno, N. et al. Remodeling of lipid vesicles into cylindrical micelles by $\alpha-$ synuclein in an extended $\alpha$-helical conformation. J. Biol. Chem. 287, 29301-29311 (2012).

35. Varkey, J. et al. $\alpha$-synuclein oligomers with broken helical conformation form lipoprotein nanoparticles. J. Biol. Chem. 288, 17620-17630 (2013).

36. Walter, A., Vinson, P. K., Kaplun, A. \& Talmon, Y. Intermediate structures in the cholate-phosphatidylcholine vesicle-micelle transition. Biophys. J. 60, 1315-1325 (1991).

37. Peter, B. J. et al. BAR domains as sensors of membrane curvature: the amphiphysin BAR structure. Science 303, 495-499 (2004).

38. Boucrot, E. et al. Membrane fission is promoted by insertion of amphipathic helices and is restricted by crescent BAR domains. Cell 149, 124-136 (2012).

39. Andelman, D., Kozlov, M. M. \& Helfrich, W. Phase transitions between vesicles and micelles driven by competing curvatures. EPL 25, 231-236 (1994).

40. Lichtenberg, D., Ahyayauch, H., Alonso, A. \& Goñi, F. M. Detergent solubilization of lipid bilayers: a balance of driving forces. Trends Biochem. Sci. 38, 85-93 (2013).

41. Ford, M. G. J. et al. Curvature of clathrin-coated pits driven by epsin. Nature 419, 361-366 (2002)

42. Gallop, J. L. et al. Mechanism of endophilin N-BAR domain-mediated membrane curvature. EMBO J. 25, 2898-2910 (2006)

43. Betancourt-Solis, M. A., Desai, T. \& McNew, J. A. The atlastin membrane anchor forms an intramembrane hairpin that does not span the phospholipid bilayer. J. Biol. Chem. 293, 18514-18524 (2018).

44. Bian, X. et al. Structures of the atlastin GTPase provide insight into homotypic fusion of endoplasmic reticulum membranes. Proc. Natl Acad. Sci. USA 108, 3976-3981 (2011).

45. Liu, T. Y. et al. Cis and trans interactions between atlastin molecules during membrane fusion. Proc. Natl Acad. Sci. USA 112, 1851-1860 (2015).

46. Byrnes, L. J. et al. Structural basis for conformational switching and GTP loading of the large G protein atlastin. EMBO J. 32, 369-384 (2013).

47. Liu, T. Y. et al. Lipid interaction of the $\mathrm{C}$ terminus and association of the transmembrane segments facilitate atlastin-mediated homotypic endoplasmic reticulum fusion. Proc. Natl Acad. Sci. USA 109, 2146-2154 (2012).

48. Faust, J. E. et al. The atlastin C-terminal tail is an amphipathic helix that perturbs the bilayer structure during endoplasmic reticulum homotypic fusion. J. Biol. Chem. 290, 4772-4783 (2015).

49. Twomey, E. C. et al. Substrate processing by the Cdc48 ATPase complex is initiated by ubiquitin unfolding. Science 365, eaax1033 (2019).

50. Strop, P. \& Brunger, A. T. Refractive index-based determination of detergent concentration and its application to the study of membrane proteins. Protein Sci. 14, 2207-2211 (2005).

51. Bligh, E. G. \& Dyer, W. J. A rapid method of total lipid extraction and purification. Can. J. Biochem. Physiol. 37, 911-917 (1959).

52. Wang, S., Romano, F. B., Field, C. M., Mitchison, T. J. \& Rapoport, T. A Multiple mechanisms determine ER network morphology during the cell cycle in Xenopus egg extracts. J. Cell Biol. 203, 801-814 (2013).

53. Wang, S., Romano, F. B. \& Rapoport, T. A. Endoplasmic reticulum network formation with Xenopus egg extracts. Cold Spring Harb. Protoc. 2019, pdbprot097204 (2019).

54. Zivanov, J. et al. New tools for automated high-resolution cryo-EM structure determination in RELION-3. Elife 7, e42166 (2018).
55. Aoki, K., Nakajima, R., Furuya, K. \& Niki, H. Novel episomal vectors and a highly efficient transformation procedure for the fission yeast Schizosaccharomyces japonicus. Yeast 27, 1049-1060 (2010).

56. Lee, I. J. et al. Factors promoting nuclear envelope assembly independent of the canonical ESCRT pathway. J. Cell Biol. 219, e201908232 (2020).

57. Gietz, R. D. \& Schiestl, R. H. High-efficiency yeast transformation using the LiAc/SS carrier DNA/PEG method. Nat. Protoc. 2, 31-34 (2007).

58. Wang, N. et al. The novel proteins Rng8 and Rng9 regulate the myosin- $\mathrm{V}$ Myo51 during fission yeast cytokinesis. J. Cell Biol. 205, 357-375 (2014).

\section{Acknowledgements}

We thank Snezhana Oliferenko, Sophie Martin, I-Ju Lee, Jian-Qiu Wu, and Susan Wente for sharing yeast strains and reagents; the Nikon Imaging Center, the Center for Macromolecular Interactions, and the Electron Microscopy Facility at Harvard Medical School for technical assistance; and Bradley French, and Yoko Shibata for critical reading of the manuscript. L.D.C was supported by a fellowship from the American Heart Association (20POST35140000), Y.G. by a fellowship from the Damon Runyon Cancer Research Foundation (DRG-[2354-19]), M.M.K by the Israel Science Foundation (3292/ 19) and the Deutsche Forschungsgemeinschaft (DFG) (SFB 958), and T.S. by the Israel Science Foundation (2751/20). T.A.R. is a Howard Hughes Medical Institute investigator.

\section{Author contributions}

N.W. designed and performed essentially all in vitro and yeast experiments; L.D.C purified REEP fusions and performed EM experiments; Y.G. collected cryo-EM images; N.W., L.D.C., Y.G., and T.A.R. analyzed the data; M.M.K., T.S., and T.A.R. interpreted the data and wrote a draft of the paper. All authors contributed to the writing of the paper.

\section{Competing interests}

The authors declare no competing interests.

\section{Additional information}

Supplementary information is available for this paper at https://doi.org/10.1038/s41467020-20625-y.

Correspondence and requests for materials should be addressed to T.A.R.

Peer review information Nature Communications thanks Patricia Bassereau, Jason Schnell and the other, anonymous, reviewer(s) for their contribution to the peer review of this work. Peer reviewer reports are available.

Reprints and permission information is available at http://www.nature.com/reprints

Publisher's note Springer Nature remains neutral with regard to jurisdictional claims in published maps and institutional affiliations.

Open Access This article is licensed under a Creative Commons Attribution 4.0 International License, which permits use, sharing, adaptation, distribution and reproduction in any medium or format, as long as you give appropriate credit to the original author(s) and the source, provide a link to the Creative Commons license, and indicate if changes were made. The images or other third party material in this article are included in the article's Creative Commons license, unles indicated otherwise in a credit line to the material. If material is not included in the article's Creative Commons license and your intended use is not permitted by statutory regulation or exceeds the permitted use, you will need to obtain permission directly from the copyright holder. To view a copy of this license, visit http://creativecommons.org/ licenses/by/4.0/

(C) The Author(s) 2021 